NASA Technical Memorandum 88947

AIAA-87-0530

\title{
Structureborne Noise Control in Advanced Turboprop Aircraft
}

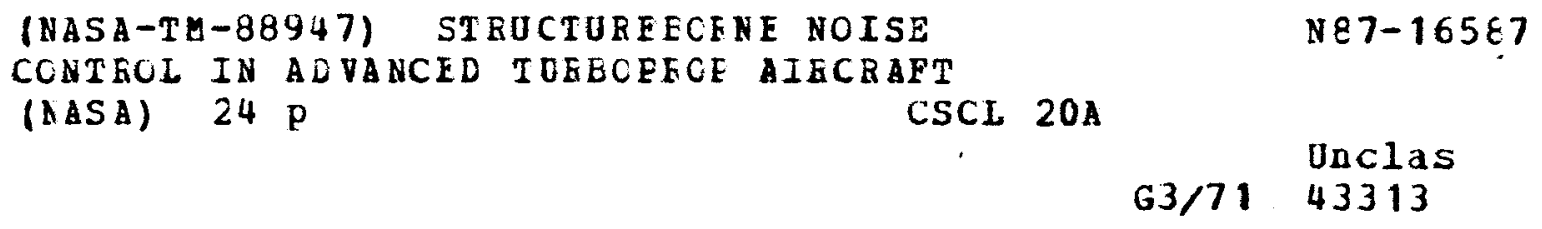

Irvin J. Loeffler

Lewis Research Center

Cleveland, Ohio

Prepared for the

25th Aerospace Sciences Meeting

sponsored by the American Institute of Aeronautics and Astronautics Reno, Nevada, January 12-15, 1987 
by

Irvin 1. Loeffler

National Aeronautics and Space Administration

Lewis Research Center

Cleveland, Ohio 44135

\section{Abstract}

Structureborne noise is discussed as a contributor to propeller aircraft interior noise levels that are nonresponsive to the application of generous amounts of cabin sidewall acoustic treatment. High structureborne noise levels may jeopardize passenger acceptance of the fuelefficient high-speed propeller transport aircraft designed for cruise at Mach 0.65 to 0.85 . These single-rotation tractor and counter-rotation tractor and pusher propulsion systems will consume 15 to 30 percent less fuel than advanced turbofan systems.

Structureborne noise detection methodologies and the importance of development of a structureborne noise sensor are discussed. A structureborne noise generation mechanism is described in which the periodic components of propeller swirl produce periodic torques and forces on downstream wings and airfoils that are propagated to the cabin interior as noise. Three concepts for controlling structureborne noise are presented: (1) a stator row swirl remover, (2) selection of a proper combination of blade numbers in the rotor/stator system of a single-rotation propeller, and the rotor/rotor system of a counter-rotation propeller, and (3) a tuned mechanical absorber.

\section{Introduction}

Propeller-driven aircraft are characterized by noise and vibration levels which in many cases adversely affect the comfort of passengers and crew. Generous amounts of acoustic treatment applied to the fuselage sidewalls have often been ineffective in reducing the interior noise to desired levels, indicating a probable noise floor due to an alternate propaqation path. As indicated in Fig. 1, externally generated noise from the propeller and engine propagates through the air and excites the cabin sidewalls, which then radiate noise to the cabin interior. However, vibrations from the propeller, engine, gearbox, and propeller wake propagate through the wing structure and the consequent excitation of the $c$ abin sidewalls causes them to radiate noise to the cabin interior. This "structureborne no ise" (SBN) appears to be a prime contributor to interior noise levels that are nonresponsive to the conventional means of suppressing airborne noise $(A B N)$.

The current world-wide interest in energy conservation has made the advanced turboprop, a high-speed propeller also known as a propfan, a strong candidate as a propulsor for transport aircraft in the 1990's and beyond.1,? Both sinqlerotation (SR) and counter-rotation (CR) high speed propeller propulsion systems are being developed to cruise at flight speeds of Mach 0.65 to 0.85 . These systems are expected not only to match the flight speeds of the 1980 vintage turbofan fleet, but to operate on 50 to 60 percent less fuel. They will consume 15 to 30 percent less fuel than advanced turbofan engines.

A model of an SR tractor propfan is shown in Fig. 2. A wing-mounted installation of this propeller is pictured in Fig. 3. The General Electric UDF (unducted $f a n$ ) engine is a unique gearless $C R$ pusher high-speed propeller system designed for aft mounting, as shown in Fig. 4. A geared CR tractor propeller system suitable for winq-mounting is shown installed on a four-engine military transport in the illustration of Fig. 5. All three of these installations present a strong potential for unacceptably high SBN levels in the aircraft cabins.

Although high-speed propellers are designed for lowered noise with their thin highly-swept blades, the supersonic tip speed and the high blade loading of these propellers produce noise levels some $25 \mathrm{~dB}$ or more higher than those of conventional propellers. These high noise levels may require fuselage sidewall transmission loss values of 50 or $60 \mathrm{~dB}$ at a propeller blade passage frequency (BPF) in the ranqe of 150 to $300 \mathrm{~Hz}$, with somewhat less severe requirements at higher harmonics of the BPF. If the conditions that produce higher propeller $A B N$ levels also result ultimately in higher SBN levels, such levels on an advanced turboprop aircraft may well be as much as $30 \mathrm{~dB}$ above the already unacceptably high SBN levels of the conventional propeller aircraft. Furthermore, SBN technology is in its infancy, and the generation and propagation mechanisms are currently the subject of theory, conjecture, and experiment.

While SBN appears to be a major threat to passenger acceptance of the advanced turboprop commercial transport aircraft, its very existence is based largely on inference from previous unsuccessful attempts to produce a quiet interior in a propeller-driven aircraft. To the best of the author's knowledge, SBN has never been unequivocally isolated and measured in an aircraft in flight. This is due to the difficulties in separating the causes of excitation of the interior vibrating surfaces--how much was induced by external ABN impingement and how much by structureborne transmission.

In the early 1980's, while responsible, in cooperation with the Aircraft Noise Reduction Division of the Langley Research Center, for planning the interior noise acoustic research program for the Lewis-managed Advanced Turboprop Project, the author espoused a number of concepts involving SBN research, as qiven below:

(1) Laboratory model tests and ground based tests on propeller aircraft are very valuable even though neither closely simulates inflight SBN conditions. 
(2) The wake of an SR propeller impinging on a downstream wing, pylon, or tail surface produces a periodic torque load that can propagate to the cabin interior as SBN.

(3) A stator row downstream of an SR propeller offers a means of reducing SBN.

(4) The selection of an appropriate combina$t$ ion of blades in an SR rotor/stator system can reduce SBN transmitted to an aircraft interior. This concept has been expanded in this paper to also apply to CR propeller systems.

(5) Strain gauges mounted to the wing spars near the wing roots should be investigated as SBN sensors.

This paper will serve to document these concepts and to cite some of the current pertinent SBN research activity. The Structural Acoustics Branch of the Langley Research Center is currently managing an extensive in-house and contract research program investigating these and other concepts related to SBN in propeller-driven aircraft. The unique problems associated with SBN measurement, the feasibility of various experimental and analytical approaches in SBN research, a mechanism for SBN generation as a result of propeller swirl impingement on downstream airfoils, and possible methodologies for reducing SBN in propeller-driven aircraft will be discussed in subsequent sections of this paper.

\section{Structureborne Noise Detection}

\section{Separation of ABN and SBN Signals}

In general, propeller-generated SBN cannot be directly measured in the cabin of an aircraft in flight. As indicated in Fig. 6 the cabin interior noise level consists of both $A B N$ and SBN. Because of the very high correlation between propeller $A B N$ and SBN (the spectrum of each consists primarily of the propeller blade passage tone and its harmonics) a microphone in the cabin measures the combined acoustic levels at each propeller tone frequency, and the SBN contribution is indiscernable from the $A B N$ contribution. Direct measurement of SBN requires some means of separating SBN from the $A B N$ signal.

\section{Acoustic Barrier Technique}

One way to directly measure the $S B N$ is by reducing the $A B N$ to a level well below that of the SBN level, as with a barrier technique as shown in Fig. 7.

Two examples of application of the barrier technique are:

(1) A wall to block the airborne noise path

(2) An enclosure to completely seal off the receiver (cabin exterior) from the $A B N$ source

An approach, developed by William Mayes of the $L$ angley Research Center and the author in an SBN ground experiment conducted at Langley on a DeHavilland Twin Otter aircraft, 3 employed the second of the two barrier techniques. Interior noise from the propeller was measured in the unaltered (baseline) state, and again with one and then two layers of acoustic material wrapped around the fuselage. This approach is based on the principle that known increases in sidewall acoustic attenuation produce a predictable change in the $A B N$ level without a significant effect on SBN. Thus, if point (1) in Fig. 8 is a baseline condition, increasing attenuation results in points (2) to (4). Without SBN points (3) and (4) would fall on the airborne noise curve at (3') and $\left(4^{\prime}\right)$, respectively. As the ABN level is lowered below the SBN level, the curve becomes asymptotic to the horizontal line representing the SBN level. The line is horizontal because $S B N$ is invariant with $A B N$ barrier treatment level. One problem with this approach is that the acoustic material wrapped around the fuselage may influence SBN propagation in addition to blocking $A B N$.

While it is possible, in principle, to measure SBN by the brute force method described above, in a given situation it must be physically possible and feasible to suppress the $A B N$ to a level below that of the SBN, and to accurately predict or measure the various attenuation levels. Attenuation is most readily predictable in the mass-law frequency range, where for single wall treatment the transmission loss at a given frequency varies with the logarithm of the treatment weight per unit surface area.

\section{SBN Vibration Path Interruption}

It is also theoretically possible to determine SBN by eliminating the SBN component from the measured interior noise. The level with SBN removed is subtracted antiloqarithmically from the combined $A B N+S B N$ level. The remainder is the SBN level. This method, however, $c$ an be used to measure structureborne noise only when the structure borne noise level is approximately equal to or hiaher than the airborne noise. For current vintage propeller-driven airplanes this is qenerally not the case. This approach could best be used in conjunction with the testing of $A B N$ acoustic treatment to ensure that the measured noise levels are uncontaminated by the presence of SBN in the total noise level. The method has, perhaps, one other application. It can be used to demonstrate that the structureborne noise component is not dominant in those cases where noise measurements with and without the structureborne path broken are substantially equal. Examples of the use of this method that have been suggested are (1) disconnecting the wing or pylon supporting an operating engine (providing the tail empennage is outside the propeller slipstream), (2) dismounting and separately supporting the engine/propeller system, and (3) locating an equivalent engine/propeller system (perhaps another airplane) near the test aircraft fuselage. Methods (2) and (3) can also test propeller wake effects on a wing or pylon by using forward and reverse pitch or by shifting the engine/propeller axis by $180^{\circ}$.

An example of breaking the SBN propagation path by method (1) would be by detaching the fuselage from the wings of a North American Rockwell OV-10 Bronco, shown in Fig. 9, by removing the four attachment bolts leaving the powerplants, wings, and tail empennage undisturbed. The lower part of the figure shows the measured wing and vibration mode at $201.5 \mathrm{~Hz}$, which will be discussed in a later section of this paper. 


\section{Inflight Measurement of SBN}

Measurement of SBN in flight presents even greater difficulties. ABN suppression methods such as the barrier techniques are impractical due to the imposition of significant added weight to the aircraft, unacceptable drag, the inability of the structure to withstand flight aerodynamic forces, and the effect of the barrier on aircraft flight control and safety. Thus, practical considerations require that barrier experiments be conducted as ground tests. It may be argued that the stresses imposed in an aircraft resting on its 1 anding gear under ground static conditions are quite different from those imposed in the same a ircraft in wing-supported flight conditions. Nevertheless, it may likewise be argued that if the physics of SBN generation, propagation, and control $c$ an be demonstrated and understood on an aircraft on the ground, it is likely that the principles can also be applied to an aircraft in flight. Consequently, it appears that ground tests provide a valuable and necessary means for studying SBN in full scale aircraft. Furthermore, it is also recognized that the source noise provided by an operating propeller in static conditions on the ground differs greatly from that of the same propeller in flight. What is needed is a sensor or measurement technique that is capable of measuring SBN directly, but which is insensitive to $A B N$. Such a device would provide a capability of evaluating the effectiveness of proposed SBN control devices on an aircraft in flight. The development of such a sensor technique may be the real key to success in the development of SBN control technology for propeller transport aircraft in flight.

\section{Structureborne Noise Generation}

\section{SBN Generation Mechanisms}

Any mechanism by which time-varying forces $c$ an be transmitted by the engine/propeller system to the aircraft structure is a possible contributor to SBN. Mechanical imbalance of the enginel propeller rotating system is one obvious source of vibration transmission to the engine mounting system Another is nonuniform or off-axis inflow to the propeller that produces a variation of lift and drag forces on each blade during one rotation of the propeller. In addition blade-to-blade variations in lift and drag can yield one-per-rev harmonic variations in propeller torque, thrust, and lateral forces. All of these forces are transmitted through the engine and propeller mountings, and the tuning of these mountings to absorb the attendant vibrations could be one step in reducing these vibrations. This method was used by Unruh ${ }^{4}$ to reduce SBN by $10 \mathrm{~dB}$ in a single-engine light aircraft.

In one instance the unacceptably high interior noise and vibration of is small propellerdriven business aircraft was greatly reduced when the aircraft was converted from a conventional tail empennage to a T-tail where the horizontal stabilizer and elevator system was relocated outside the propeller slipstream, an early indication of SBN due to the action of propeller wake.

In their structureborne noise experiment Metcalf and Mayes ${ }^{3}$ concluded that the structureborne noise measured in a ground test of a Dehavilland Twin 0tter was caused by propeller blade wake and tip vortex interaction with the wing. The barrier method discussed in the previous section was used to isolate the SBN by two wrappings of the fuselage in acoustic material. The SBN level was determined to be 10 or 11 below the $A B N$ level. Application of a wrap on the wing in the propeller wake region reduced the SBN level by 6 or $7 \mathrm{~dB}$, and gave confidence that SBN was, indeed, isolated. A mechanism will now be described by which the propeller wake and tip vortex produce noise and vibration in the cabin interior of wing-mounted propeller-driven airplanes such as the Twin Otter.

\section{Propeller Wake SBN Generation}

An SBN qeneration mechanism unique to propeller-driven aircraft is provided by the reaction of a downstream airfoil to an upstream propeller wake. For aircraft driven by singlerotation wing mounted tractor propellers, a wing or tail surface located downstream of a propeller receives a periodic torque loading at the propeller blade passage frequency and its harmonic due to the unsteady flow produced by the individual propeller blades. This periodic torque produces a "wiggle," a small periodic flexing of the airfoil which is attached to the fuselage structure. The resultant flexing of the fuselage results in the radiation of sound to the cabin interior for periodic torques in the audible frequency range. If the center of the propeller slipstream passes above or below the airfoil, or if the airfoil is swept, there will also be net periodic upward and downward forces on the airfoil. If present, these forces also contribute to a "wiggle" in the downstream airfoil.

Most of the flow behind a propeller typically follows a helical or swirling path, as shown in Fig. 10. However, the detailed flow pattern throughout the propeller disk is quite complex, as shown in the calculated SR-3 propeller wake secondary flow plot of Fig. 11 taken from Ref. 5. Considerable variation in magnitude and direction is evident. The tangential component of the flow is clockwise (CW) at some points and counterclockwise (CCW) at others. While the spanwise flow is mostly toward the tip, in the tip vortex region the flow is toward the hub on the blade suction side of the wake. The nonuniformity in the tangential direction (circumferential) is, of course, cyclical; the pattern repeats from blade to blade. In Fig. 11 the blade wake locations are evidenced by the propeller tip vortex flow as well as the pronounced chanqe in spanwise flow on opposite sides of the blade. In the midspan and hub region where the solidity is high, the flow is characteristic of cascade or channel flow, while in the tip region the flow is more representative of isolated airfoil flow, although this is also due to the influence of the tip vortex.

In the propeller wake plot of Fig. 11, it may be assumed that the center of the vortex is located in the vicinity of the propeller blade tip. In the region lying well inside the blade tip region the flow undergoes only small changes in magnitude and direction; in the blade tip region the changes are rather severe. Between the blade wakes the flow near the tip has only a small swirl component. 
It may be useful to consider the swirl flow as having two tangential components; a constant nontime-variant component analogous to a DC current flow, and an oscillating or periodic time-variant component analogous to an $A C$ current flow, as illustrated in Fig. 12.

Physical evidence of significant periodic tangential forces introduced in the propeller wake at blade tip distance from the propeller shaft has been obtained. Miller, Dittmar, and Jerack $i^{6}$ measured these oscillating (AC) forces in terms of the pressures imposed on sensors mounted on opposite sides of a nonlifting airfoil which was traversed radially in a plane one-propeller diameter downstream of the propeller plane, as shown in Fig. 13. R.M.S. dynamic pressure traces for the advancing propeller vane side are given in Fig. 14 as a function of radial distance from the propeller axis for wind tunnel flow Mach numbers of 0.6 and 0.8. It is quite evident that the largest periodic pressures are associated with the propeller tip vortex region.

For a propeller mounted with its axis of rotation on or near the wing chord line, one effect of the swirl flow in Fig. 11 is to impose a steady or $D C$ twisting force or torque on the downstream wing, tending to rotate it in the vertical plane in the same rotational direction as the propeller. In addition, there is an unsteady or AC torque tending to cause an oscillating rotation of the wing around the propeller axis, as illustrated in Fig. 15. Figure 15 illustrates the motion of the wing associated with the first order mode of flexing or forced vibration of the wing. If this torque were constant with time, i.e., with the $D C$ component only, it would pose no problem with respect to cabin interior noise and vibration. However, as will be discussed in detail later, the pressure at any point in the propeller wake, such as a point on the wing surface, varies with time during a cycle. The period of the cycle, T, is the time required for the pattern of $\mathrm{Fig} .11$ to repeat. Since the pattern rotates at the prope1ler rotational speed, each blade wake passes any given point at the propeller blade passage frequency, or BPF Hence,

$$
T=\frac{1}{\mathrm{BPF}}
$$

and

$$
B P F=B N
$$

where $B$ is the number of propeller blades and $\mathrm{N}$ is the propeller rotational speed in revolutions per second.

In addition to the bending modes of the wing it is also likely that torsional modes (spanwise twisting) will also be excited.

An example of this kind of motion is shown in Fig. 9 of the vibration mode of the NASA Lewis OV-10 North American Rockew 11 Bronco aircraft as measured by Langley. ${ }^{7}$ Here the $r$ ight wing is excited by a white noise signal from a shaker applied to the right wing. The nodal line at midspan is at the engine/propeller location. The mode shown is for $201.5 \mathrm{~Hz}$, approximately twice the BPF of the propeller at cruise speed. Many other modes were also measured, many of them involving torsional vibration, or a twisting motion of the wing about a spar or a leading or trailing edge. With reference to $\mathrm{Fig} .15$ it would seem that strain gauges mounted near the top and bottom of the wing main spars near the wing root should be a preferred choice for a sensor to detect the flexural or bending wave in the wing as shown. The fuselaqe structure must react directly to the forces imposed upon it at the wing attachment point. Consequently, the details of wing deformation or motion away from the wing root are probabiy of little significance. Therefore the dynamic strain gauge near the root senses the periodic strain, probably a good measure of the vibration level reaching the fuselage. Additional combinations of gauges mounted fore and aft $c$ an be used to sense the torsional strain at the wing root.

The main objective of the Langley OV-10 research effort was to determine the wing and fuselage dynamic response of a full scale aircraft to a variety of excitation modes. The wing was excited by impact hammer, by single and by multiple phased shakers, and by the propeller itself. Motion of the wing and fuselage was detected by accelerometers and strain gauges. One of the objectives was to determine appropriate locations for SBN sensors. From Fig. 9 it is quite evident that a nodal point on the wing would not be a good location for an accelerometer intended for sensing wing vibration response at a frequency of $201.5 \mathrm{~Hz}$.

The development of an SBN sensor or SBN detection methodology is one of the objectives of the laboratory model SBN experimental research program conducted by Unruh ${ }^{8}$ of the Southwest Research Institute, currently in progress. This work is being done under contract to Langley. Unruh's test uses a $102 \mathrm{~cm}$ (40 in.) diameter fuselage model enclosed in a $165 \mathrm{~cm}$ (65 in.) diameter concrete pipe to provide $A B N$ shielding. A propeller, an impact hammer, a speaker, and possibly phased shakers will be used to excite the model wing attached to the fuselage and protruding through a sealed opening in the ABN shield. Ground-based transfer function measurements relating input forces to wing root responses by accelerometers and strain gauges and to fuselage interior noise levels will be used to develop a method for measuring SBN inflight.

Propeller Periodic Torque Load on Downstream Airfoil

The experimental determination of the torque loading on the wing at a given instant would require a distribution of th instantaneous pressures with time for a suitable grid covering the wing surfaces exposed to the propeller wake. Then, for each selected time, the integrated product of the pressure, the grid area, and the distance from the propeller axis can be algebraically summed to yield a net torque acting on the wing. From these values the time history of the net torque for a complete period can be determined.

Since an experimental study involves difficulties in providing a sufficient number of sensors to obtain even a modest resolution of the unsteady pressure distribution on the wing behind a propeller and also the need for extremely accurate phase information, an analytical study was included in the Langley SBN program. The analys is 
by Weir ${ }^{9}$ readily accommodates the parametric investigation of such variables as propeller location with respect to the wing, propeller thrust, propeller blade number, wing sweep and taper, and advance ratio. Test cases computer for the Twin 0tter, the OV-10 Bronco, and the NASA PTA aircraft showed significant differences in the temporal variation of the spanwise lift distribution and the wing root moment. The analysis system is available for analysis of wing structural response.

An effort to completely model the SBN generation and propagation process is currently underway by the Cambridge Acoustical Associates. This Small Business Innovative Research contract work is managed by the Langley Research Center. To obtain experimental data to guide the effort, input and transfer impedances of the wing and fuselage structures of the NASA-Langley Beechcraft Baron 58P laboratory test airframe were measured by Cole and Martini.10 Engines and fuel were not present for the tests. Structural-acoustic transfer functions of the fuselage both with and without attached wings, structural input impedance measurements, and structural-acoustic crosscorrelations were obtained. The transfer functions between cabin pressure and excitation force applied to the structure indicated substantial variability with frequency, with no tendency to become asymptotic with higher frequency. Furthermore, the dynamics of the aircraft structuralacoustics systems provides close coupling of the acoustic space in the cabin to many degrees of freedom of the structure. These findings indicate a probable difficulty in "fine tuning" a structure to minimize cabin response at propeller tone frequencies.

\section{SBN Propagation in Wing or Pylon}

From an acoustics viewpoint, the elastic wave of most interest in a structure is the bending or flexural wave, a combination of compressional and shear waves.11 Because of the large transverse displacements associated with these waves, they readily couple to compressional waves in surrounding fluids. Thus, bending waves are easily excited in a structure such as a wing or fuselage by compression waves in a surrounding medium, and the structure is likewise capable of exciting compression waves in a surrounding medium. However, Langley tests with strain gauges on the main spars of the OV-10 indicated that a pure compression wave in the main wing spar may also contribute to the propagation of $S B N$ through a wing to the fuselage. While the velocity of a compressional wave in a bar or plate is invariant with frequency, the velocity of the bending or flexural wave varies with the square root of the frequency. Thus, the propagation process is dispersive, and the wave form is not preserved. Considerable research is indicated to properly define the precise nature of the propagation of SBN in aircraft structures.

\section{SBN Generation by Counter-Rotation Propellers}

For counter-rotation propellers the aft blade row operating in the circumferentially-varying swirl from the upstream blade row experiences a cyclic or periodic variation in torque and thrust loading determined by the passage of each downstream blade through the wake of each upstream blade. The periodic loading of the downstream rotor results in periodic torque and thrust which would ordinarily be carried by the propulsion system mounting and ultimately by the main structure of the aircraft. Thus, the CR propeller may also function as an SBN generator. However, for the GE/NASA UDF engine with the two counter-rotating blade rows carried by free turbines, strong per $i-$ odic reaction torques should not reach the engine mount. A periodic thrust, however, may provide a source of SBN generation.

\section{SBN Control Methodology}

\section{SBN Control Concepts}

A variety of SBN control concepts for propeller-driven aircraft appear to have some potential. Among these are:

(1) Stator row swirl remover

(2) Blade number combination selection

(3) Tuned mechanical absorber

(4) Tuned wing/fuselage (or pylon/fuselage) attachments

(5) Structural damping

(6) Compliant airfoil surfaces

Only the first three will be discussed in this paper.

\section{Stator Row Swirl Remover}

The first proposed device consists of a number of radially oriented turning vanes somewhat like a propeller that remains stationary instead of rotating. These turning vanes or stators are mounted behind the propeller and are designed with blade element shape and camber to efficiently turn the incoming flow from the propeller to the axial direction upon leaving the turning vane as shown in Fig. 16. The vanes acting together thus remove th4 swirl from the propeller exit flow.

As mentioned earlier, if the swirl flow behind the propeller were to have constant magnitude and direction, it would present no problem with respect to structureborne noise. However, at any given radial distance from the propeller axis, the flow has a constant or $D C$ component and a varying or $A C$ component. In fact, it is not necessary to remove the constant or $D C$ swirl, the component imposing a constant torque force on the wings, in order to eliminate structure-borne noise due to swirl interaction with the wings. A stator row system designed to function solely as a flow straightening device, such as the flow straightening honeycomb screens in a wind tunnel, would be quite adequate. However, such a stator system provides a very attractive means to a iso remove the DC swirl component, and thereby increase the propeller propulsive efficiency.

To be effective in eliminating the $A C$ swirl component from the propeller exit flow the downstream stator row must have a reasonable high solidity from hub to tip. Optimum values for a given design, of course, must be determined by analys is and experiment. The requirement for flow straightening (removing the $A C$ component swirl) is, indeed, a function of the structureborne noise reduction required in terms of the baseline structureborne noise level, the desired final interior noise level, and the benefit provided by other means of structureborne noise reduction. 
Performance and Acoustic Benefits of Stators for SR Propellers. The use of stators to efficiently recover the swirl energy from the propeller exit flow provides highly significant additional benefits. For high speed, high performance aircraft such as currently being considered in the advanced turboprop aircraft concept, the propeller swirl results in lower propeller efficiency due to kinetic energy lost in the swirl flow, complicated nacelle and wing contouring requirements to minimize drag, complicated canted engine inlet designs due to off axis flow entering the inlet, and low speed stability and control problems due to nonsymmetrical wing contours aft of the engines.

In a parametric design study of counterrotation propellers for advanced turboprop aircraft, 13 the propulsive efficiency of a system with seven stators located downstream of a tenblade single-rotation propeller was calculated. The propeller efficiency went from 80 to 87 percent. This value was only 1.1 percent point below the efficiency calculated for the recommended counter-rotation design of two counter-rotating propellers with five propeller blades each.

Some advantages of the proposed stator structureborne noise device are as follows:

(1) Reduces structureborne noise produced by the oscillating aerodynamic loading of a propeller slipstream on a wing or pylon on which it is mounted.

(2) Increases propulsion system efficiency by recovering propeller swirl energy otherwise lost.

For a wing-mounted advanced turboprop propulsion systems designed for cruise at altitudes of 25000 to $35000 \mathrm{ft}$ at flight speeds of Mach 0.6 to 0.8 , the following additional benefits can be realized:

(1) Eliminates or reduces the amount of wing (or pylon) leading edge and nacelle contouring otherwise required to achieve a low drag propulsion system installation

(2) Eliminates need for complicated canted core engine inlet design by eliminating off-axis flow into the inlet for inlet located aft of the stator

(3) Reduces low speed stability and control problems by eliminating nonsymmetrical flow over the opposite wings for same rotation direction engines on a twin or four-engine aircraft

(4) Allows the single-rotation advanced propeller (prop-fan) system to approach the propeller efficiency of an equivalent technology counter-rotation propeller system

\section{Swirl from CR Propellers}

Since a counter-rotation (CR) propeller system is designed to remove propeller swirl, such a system may seem ideally suited for a wing-mounted tractor propeller configuration with respect to structureborne noise. However, while the CR system can readily remove the $D C$ swirl component, the flow downstream of the second propeller would exhibit a relatively high level of $A C$ swirl component, particularly if the number of blades in each row is small, e.g., on the order of one-half the number of blades in an equivalent single rotation propeller design. The effectiveness of swirl removal, of either the $D C$ or $A C$ components, is determined primarily by the solidity of the downstream blade row, be it counter-rotating or fixed. For example, a single propeller blade or stator vane would process only a small portion of the total flow. A sufficient number of rotor blades or stator vanes with appropriate chord length, i.e., sufficient solidity, is required to substantially reduce the $A C$ swirl component.

\section{Blade Number Combination Selection}

SR Propellers. An important parameter to the reduction and phasing of the $A C$ swirl reaction torque is the number of stator vanes relative to the number of rotor blades. If the number of propeller blades is qiven by $B$, each downstream stator vane "sees" a peak pressure each time a propeller blade passes by, or B torque pulses per revolution. At a propeller rotational speed of $\mathrm{N}$ rps, the stator row experiences a periodic torque at a frequency of $\mathrm{BN} \mathrm{Hz}$. If the number of stator vanes is also $B$, all peak torque pulses occur simultaneousiy with a magnitude of $B$ times that of a single pulse at the blade passage frequency of $B N \mathrm{~Hz}$, the blade passage frequency. However, if the number of stator vanes is some other number $V$ rather than $B$, where $B$ and $V$ contain no common factors, then the periodic torque on the stator consists of $B V$ small pulses per revolution with a frequency of $B V N \mathrm{~Hz}$, or $V$ times the blade passage frequency.

For example, as indicated in the schematic of Fig. $17(\mathrm{a})$, if eight equally spaced stators are placed behind the 8-blade SR-3 propeller, the eight stators will each experience the reaction torque from the nearest propeller blade wake simultaneously. Since the rotor blades and stator vanes are each $45^{\circ}$ apart, when propeller blade no. 1 passes stator vane A, (see Fig. 17(a), a simultaneous passage occurs for blade no. 2 and vane $B$, and for the remaining combinations of no. 3 and $C$, no. 4 and $D$, etc. Thus, there will be a torque pulse consisting of the combined (added) torques for all eight stators for each passage of blade no. 1 with one of the stators. The corresponding reaction torque on the nacelle is represented in Fig. $17(b)$.

However, the nacelle torque reaction is very much different if nine equally spaced stators are used instead of 8 , as shown schematically in Fig. 18(a). In this case the stator vanes are oniy $40^{\circ}$ apart while the rotor blades are, as before, $45^{\circ}$ apart. Thus, at the moment the propeller wake of blade no. 1 is passing stator $A$, blade wake no. 2 is still $5^{\circ}$ away from stator $B$. After $5^{\circ}$ more of propeller rotation, or $5 / 360$ or $1 / 72$ of a revolution, blade wake no. 2 will pass stator $B$. At subsequent increments of rotation of $5^{\circ}$ or $1 / 72$ or a revolution, blade wake no. 3 will pass stator $C$, no. 4 stator $D$, etc. After one complete revolution, or $725^{\circ}$ increments later, blade wake no. 8 will have passed stator I, and 72 small torque pulses will have been imposed on the nacelle as shown in Fig. 18(b). As discussed previously, with 8 stator vanes, there were only 8 large torque pulses per revolution, as shown in 
Fig. 17(b). The large pulses from the 8-vane stator of Fig. 17(b) are also shown by the dashed lines in Fig. 18(b) for comparison. For the general case, the number of torque pulses $P$ per propeller revolution is given by the relation

$$
P=R S
$$

where $R$ is number of rotor blades, and $S$ is the number of stator vanes, and where $R$ and $S$ have no common factor. Thus, by the proper selection of $S$ for a given value of $R$, the strength of the torque reaction pulses on the nacelle supporting the stator row may be reduced by the factor $S$. Furthermore, the frequency will be multiplied by the factor $S$.

A process that would cause a shift to higher frequencies would be quite advantageous for $A B N$. Noise reduction as a function of frequency is plotted in Fig. 19 for a representative advance turboprop aircraft double-wall acoustic design. The data are from Fig. 89 of Ref. 12. In the experiment by Lockheed-Cal ifornia a $9.0 \mathrm{~m}$, $(29.5 \mathrm{ft}$ ) long section of a Swearingen Metro II aircraft was used as a 0.43 scale model of an advanced turboprop fuselage. The test article was placed in an anechoic chamber and the sidewalls were excited by simulated propeller noise and by white noise. The $3.5 \mathrm{~m}(11.5 \mathrm{ft})$ long test section was fitted with a variety of experimental high suppression wall treatments designs. As shown in the figure, increasing the BPF from 300 to $1200 \mathrm{~Hz}$, an increase by a factor of only 4, increases the noise reduction by about $30 \mathrm{~dB}$. Thus, the test double-wall reradiated $A B N$ to the cabin interior less readily at $1200 \mathrm{~Hz}$ than at $300 \mathrm{~Hz}$. If the reradiation of SBN occurs in a like manner, the shifting of the reaction torque pulse frequency to a higher frequency would be very attractive.

Even with properly designed stators the propeller exit flow will have a small oscillating swirl flow. Referring again to Fig. 11 , it is clear that as the 8-blade propeller turns the swirl pattern also rotates. Hence, a stator will be impinged by the vortex flow from one blade at one instant followed by the flow intermediately between adjacent blades and at $1 / 8$ of a revolution later, be impinged again by the vortex flow from the following propeller bladc. Consequently, the flow entering the stator will exhibit a range of incidence angles, and the turning of the flow will not be exactly to axial for all circumferential locations. The dicrepency in the tangential flow component, a variation in the stator deviation angle with a period corresponding to the time for $1 / 8$ revolution, for each spanwise location, will be quite small relative to the variation in the incidence angle for the same spanwise location. Thus, while the time averaged net swirl, the DC swirl component, will be zero or nearly zero at any spanwise location, the instantaneous values will vary from very sightly underturned to very slightly overturned during a period. As a consequence, there will be a small oscillating torque on the wing even with the stator providing zero net swirl upstream of the wing.

The chordwise flow along a given stator surface will also vary with time. The secondary flow (flow perpendicular to the propeller axis) pattern on $\mathrm{Fig} .11 \mathrm{will}$ rotate at the propeller rotational speed. Consequently, if at a given instant the stator leading edge is coincident with a vortex center of Fig. 11, at a moment later the blade leading edge will be coincident with a different portion of the flow in the CCW direction, and will ultimately be coincident with the vortex of the following blade. The stator will have processed flow for a complete cycle, i.e., from one blade to the following one. Since this is true for all stators as well as for all angular locations between stators, the flow downstream of the stator will exhibit the range of variation from blade to blade during the interval of a period and at an axial distance or wavelength of $1 / B P F \times V_{S}$, where $V_{S}$ is the velocity of the propelier slipstream flow relative to the propeller/wing system. Thus, there will remain, even in the case where no net average swirl remains, instantaneous $\mathrm{CW}$ and $\mathrm{CCW}$ swirl components which, though cancelling each other, nevertheless, introduce an oscillating pressure force on the downstream wing surface with a much reduced magnitude relative to the original unsteady swirl flow, and at the same BPF frequency as the original swirl. The further reduction of this residual oscillating swirl, if large enough to jeopardize the attainment of a desired interior noise or vibration level, can be accomplished by application of a second structureborne noise control device, the tuned mechanical absorber, to be discussed later.

CR Propellers. In a counter rotating propeller the selection of the number of forward and aft rotor blades also has an important effect on vibration transmitted to the engine/propeller system mounting, and ultimately to the fuselage itself. An approach similar to that used for single rotation propellers can be used to reduce the vibrations transmitted by a counter-rotation propeller to the mounting pylon and subsequently to an aircraft cabin. For example, consider the cyclic torque forces on the aft rotor due to the cutting of the wakes of the upstream rotor. If there are $F$ blades in the forward rotor and $F$ blades in the aft rotor, there will be $2 F$ torque pulses per revolution on the aft rotor with the rotors turning at the same rate but in opposite directions. The periodic torque load frequency on the aft rotor will be $2 F N ~ H z$. However, if the number of blades in the aft row is $A$ instead of $F$, and $A$ and $F$ contain no common factors, there will be 2FA pulses per revolution of the aft rotor with a strength of 1/A the magnitude of the pulses of the previous arrangement. The reduced strength vibrations will occur at a higher frequency of 2FAN $\mathrm{Hz}$ and may be more highly attenuated by the aircraft structure.

\section{Tuned Mechanical Absorber}

The tuned mechanical absorber is particularly suited for reducing vibratory motion in nonplatelike structures with low surface strains, and for which the vibration frequency spectrum of the response has a single resonance, a number of widely-separated resonances or a number of widely separated groups of modes. 14 It should be effective in reducing the magnitude of a flexural wave in a wing or pylon supporting an enginel propeller system.

In this approach a tuned mechanical absorber is rigidly fastened to the vibrating member at a point of high amplitude response, such as an 
anti-node. The ideal simple tuned mechanical absorber for a single degree of freedom system is illustrated in Fig. 20(a). A schematic of a typical physical arrangement of the system is shown in Fig. 20(b). In this case a viscoelastically-damped rod supports a mass to form a spring-mass oscil1 ator system. Figure 21, taken from Ref. 14, indicates that the vibrational energy removed from the system is very sensitive to frequency for lightly damped systems (low values of the damping loss factor, $n_{0}$ ). The sharply tuned and lightly damped system provides the lowest damping system weight for a single frequency response in the vibrating member. Since advanced turboprop propellers are intended to cruise at constant propeller speed (and constant blade passage frequency) the tuned absorber may be an attractive device for reducing vibration levels in a wing, pylon, or tail surface responding to the vibratory loads imposed by a propeller. Tuned mechanical absorbers can be finely tuned to the forcing frequencies, in this case, the BPF and possibly some higher harmonics.

The effectiveness of the tuned mechanical absorber has already been demonstrated in reducing vibration in an aircraft cabin from the vibration set up in the mounting of an out-of-balance engine system. Some of the early production models of the Douglas DC-9 experienced a difficulty with interior noise in the last two or three window seat positions. At these locations the cabin noise ran about $10 \mathrm{~dB}$ higher than the forward cabin noise and was dominated by two tones, one at $120 \mathrm{~Hz}$ and the other at $180 \mathrm{~Hz}$. In the cruise regime, the rotor out-of-balance vibration frequencies of interest are approximately at $120 \mathrm{~Hz}$ for the low speed rotor, and $180 \mathrm{~Hz}$ for the high speed rotor. Higher frequencies, harmonics, or vibration due to blade passage did not cause significant sound levels. However, the engine vibration levels were, in general, within specification limits. The low speed rotor includes two fan stages, four compressor stages, and three turbine stages; the high speed rotor includes seven compressor stages and a single stage turbine. A forced vibration analys is in conjunction with experiments by Douglas determined that an upward and downward motion of the engines caused an oscillating torque in the engine support structure. The forced vibration in the engine mounting and pylon structure caused a structural response in the rear bulkhead to which the engine mounts were attached. Computer analysis of the modal patterns for fuselage shell vibration for a number of frequencies of interest were calculated. The amplitude of vibration was on the order of only 0.001 in., as determined by the analysis.

Douglas engineers resolved the problem by fabricating tuned vibration absorbers, designed for 120 and $180 \mathrm{~Hz}$ resonance frequency, and attaching these to the engine supports. These devices were designed for a noise reduction of $5 \mathrm{~dB}$ for the $120 \mathrm{~Hz}$ tone and 12 to $14 \mathrm{~dB}$ for the more dominant $180 \mathrm{~Hz}$ tone. The most probably level of the loudest seat on an airplane was reduced from $102 \mathrm{~dB}$ to $97 \mathrm{~dB}$, while the upper 1 imit was reduced from 112 to $102 \mathrm{~dB}$. The "fix" was successfully applied to several other noisy DC-98 aircraft and shortly thereafter was included on production $D C-9$ 's.
Summary and Conclusions

Structureborne noise in propeller-driven aircraft has until recently received little attention from engineers and scientists. Now, however, as the advanced turboprop propeller offers a significant fuel saving over the currently popular turbofan engines at comparable flight speeds, considerable research is needed to ensure that structureborne noise will not hamper the utilization of this very attractive propulsion system.

In addition to mechanical imbalance of the rotating system and unbalanced aerodynamic forces on the propeller, the unsteady components of propeller swirl produce periodic torques on downstream airfoil surfaces that propagate to the aircraft cabin as noise. One approach to reducing structureborne noise for a single-rotation tractor propeller-driven aircraft is a specially designed stator row mounted downstream of the propeller. By proper selection of the number of stator vanes, the strength of the torque reaction forces transmitted to the fuselage can be greatly reduced and the vibration frequency $c$ an be shifted to a much higher frequency where attenuation by the aircraft structure may be more effective. Selection of the appropriate blade number combination may also reduce $S B N$ in a $C R$ propeller system.

In another approach a tuned mechanical absorber, a damped mass and spring system, is rigidly fastened to the vibrating wing or engine mounting pylon to reduce the vibration levels transmitted to the fuselage and thereby reduce the $\mathrm{SBN}$ in the cabin interior.

Application of the structureborne noise theory discussed in this paper and the proposed control methods, it is believed, can lead to greatly reduced structureborne noise in propellerdriven aircraft. In any event, the experimental and analytical investigation of the concepts discussed here should at least serve to advance current understanding of the structureborne noise phenomenon.

\section{References}

1. Dugan, J.F., Miller, B.A., Graber, E.J., and Sagerser, D.A., "The NASA High-Speed Turboprop Program," SAE Paper 801120, Oct. 1980.

2. Whitlow, J.B., Jr., and Sievers, G.K., "Fuel Savings Potential of the NASA Advanced Turboprop Program," NASA TM-83736, 1984.

3. Metcalf, V.L. and Mayes, W.H., "Structureborne Contribution to Interior Noise of Propeller Aircraft," SAE Paper 830735, Apr. 1983.

4. Unruh, J.E., and Scheidt, D.C., "Design and Test of Aircraft Engine Isolators for Reduced Interior Noise," SRI-06-4860, Southwest Research Institute, San Antonio, TX, Dec. 1982.

5. Bober, L.J., Chaussee, D.S., and Kutler, P., "Prediction of High Speed Propeller Flow Fields, Using a Three-Dimensional Euler Analysis," AIAA Paper 83-0188, Jan. 1983. 
6. Miller, B.A., Dittmar, J.H. and Jeracki, R.J., "The Propeller Tip Vortex - A Possible Contributor to Aircraft Cabin Noise," Journal of

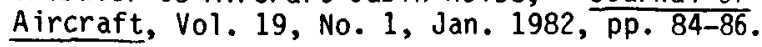

7. Clevenson, S.A., and Metcalf, V.L., "Structureborne Noise in Aircraft:Modal Tests," NASA TM-87739, 1986.

8. Unruh, J.F., "Propeller-Induced StructureBorne Noise: Laboratory-Based Test Apparatus," AIAA Paper 86-1938, July 1986.

9. Weir, D.S., "Wing Loads Induced by a Propeller Wake," AIAA Paper 86-1967, July 1986.

10. Cole, J.E., and Martini, K., "Experimental Test Results on the Beechcraft Baron 58P,

Phases I and II," Cambridge Acoustical Associates Report U-1415-349, Sept. 1986.

11. Beranek, L. L., Ed., Noise Reduction, McGrawHill, New York, 1960.
12. Prydz, R.A., Revel1, J.D., Hayward, J.L., and Balena, F.J., "Evaluation of Advanced Fuselage Design Concepts for Interior Noise Control on High Speed Propeller-Driven Aircraft." LR-30192, Lockheed-Cal ifornia Co., Burbank, CA, Sept. 1982. (NASA CR-165960)

13. Strack, W.C., Knip, G., Weisbrich, A.L., Godston, J., and Bradley, E., "Technology and Benefits of Aircraft Counter-Rotation Propellers." NASA TM-82983, 1982.

14. Rogers, L., Ed., Conference in Aerospace Polymeric Viscoelastic Damping Technology for the 1980's, AFFDL-TM-FBA-78-78, July 1978.

15. Van Dyke, J.D. Jr., Schendel, J.W., Gunderson, C.0., and Ballard, M.R., "Cabin Noise Reduction in the DC-9," AIAA Paper 67-401, June 1967. 


\section{ORIGINAL FAGE IS \\ OF POOR QUALTY}

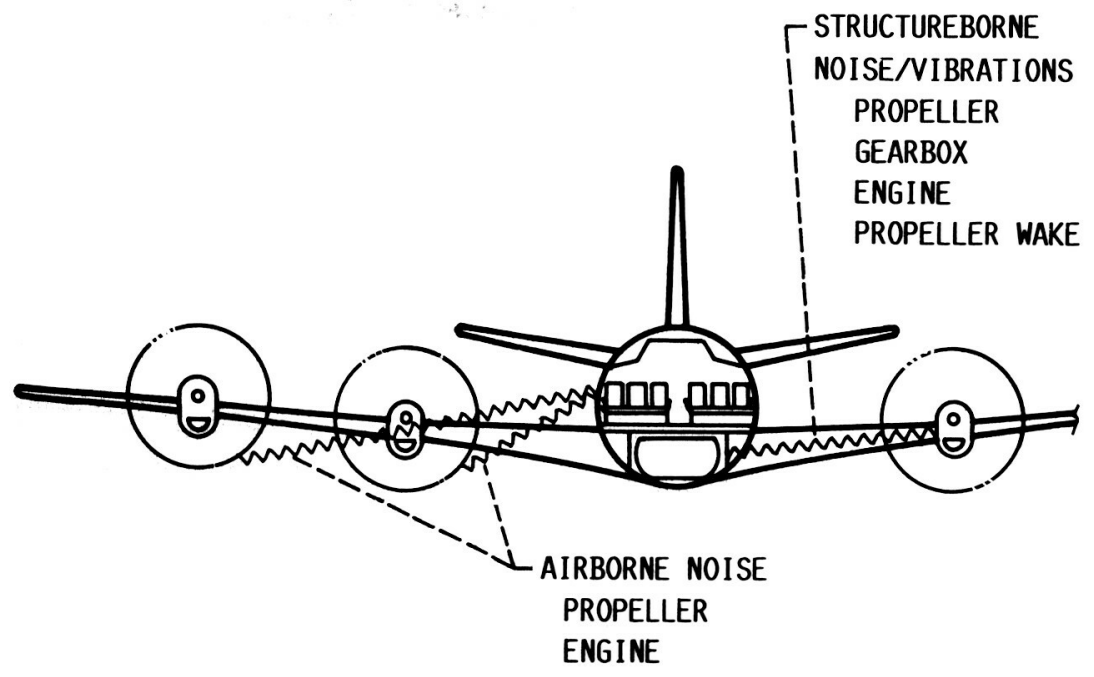

FIGURE 1.- CABIN ENVIRONMENT-AIRBORNE NOISE AND STRUCTUREBORNE NOISE.

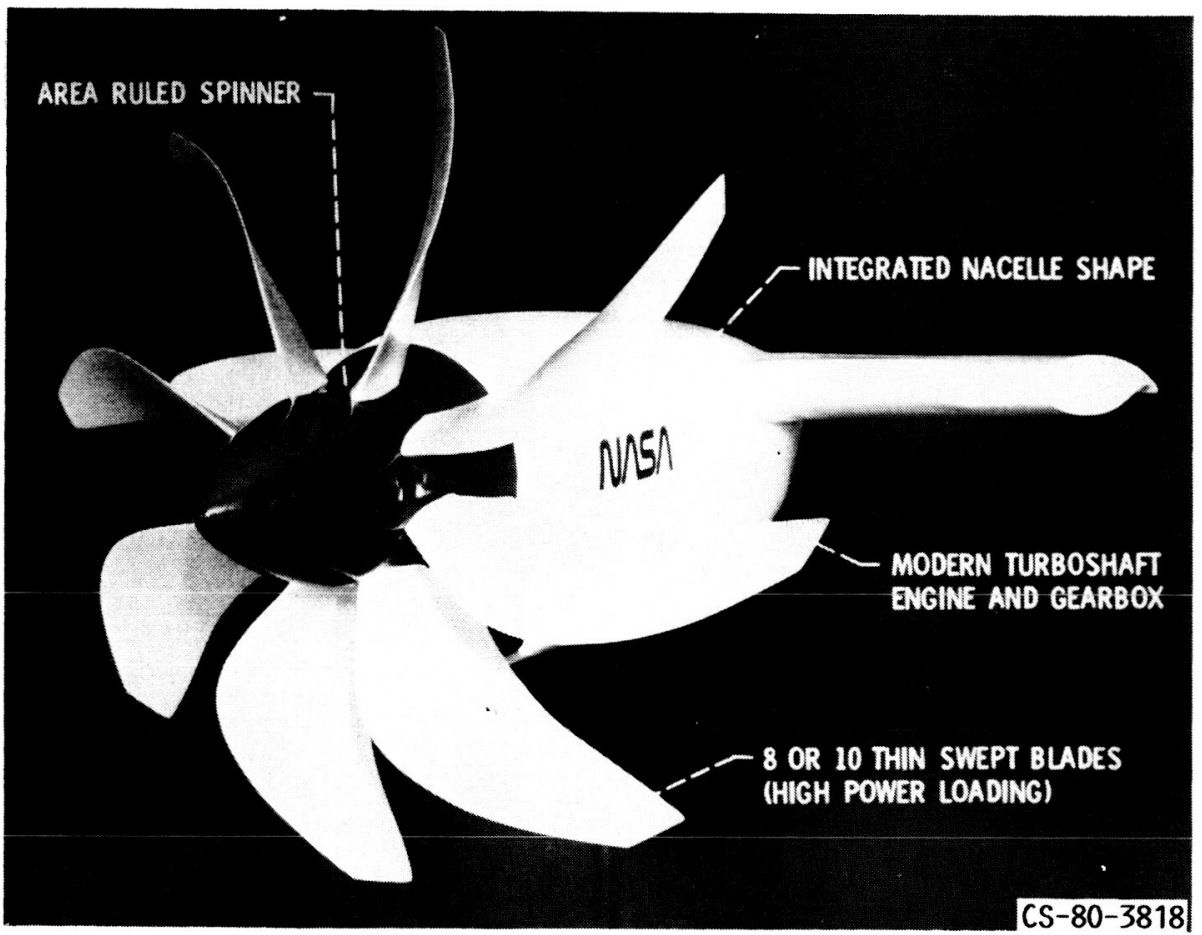

FIGURE 2. - SINGLE-ROTATION ADVANCED TURBOPROP PROPULSION SYSTEM. 
ORIGNAL PAGE IS

OF POOR QUALTY

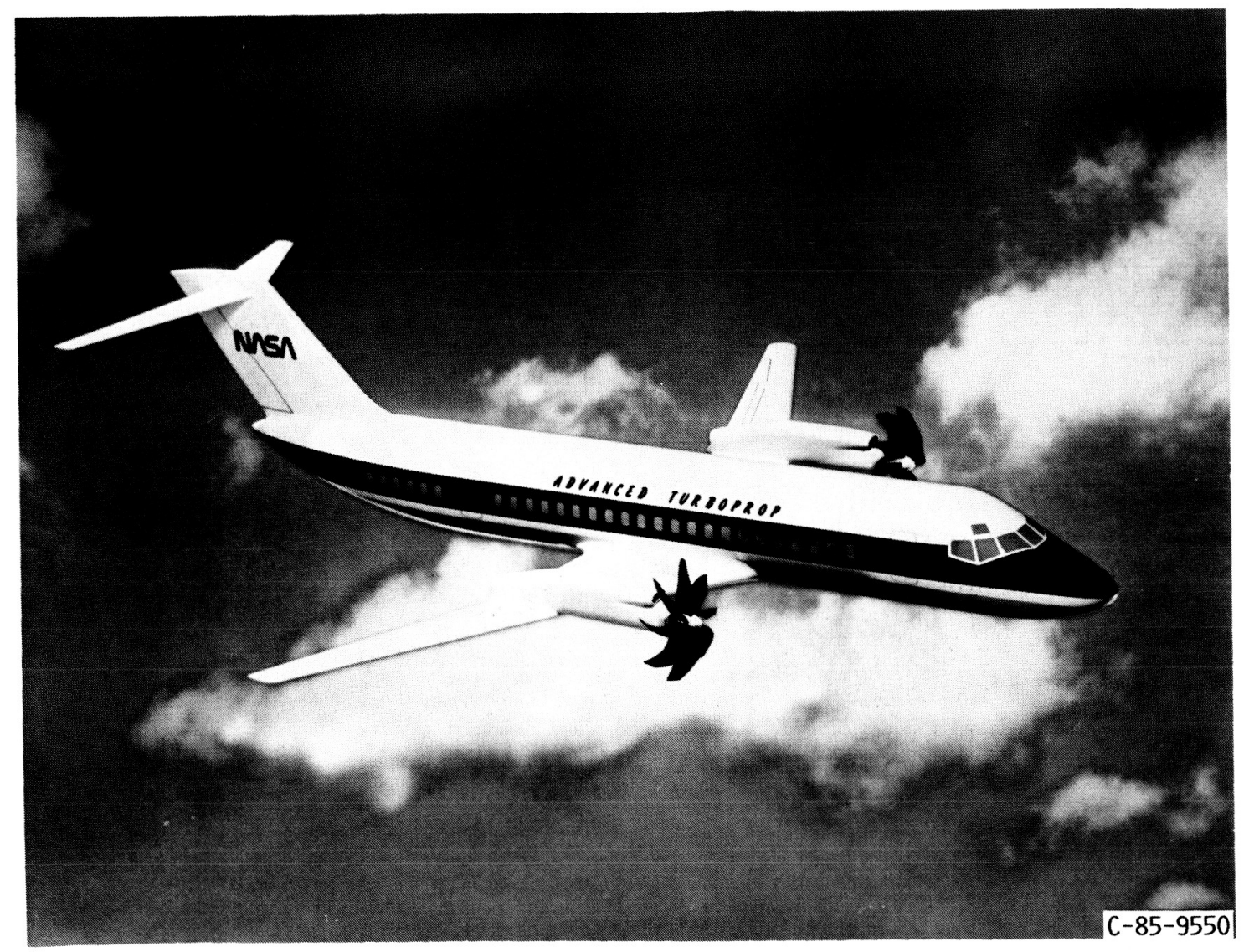

FIGURE 3. - WING-MOUNTED SINGLE ROTATION HIGH SPEED PROPELLER INSTALLATION. 
ORIGRAL PAGE IS

OF POOR QUALTY

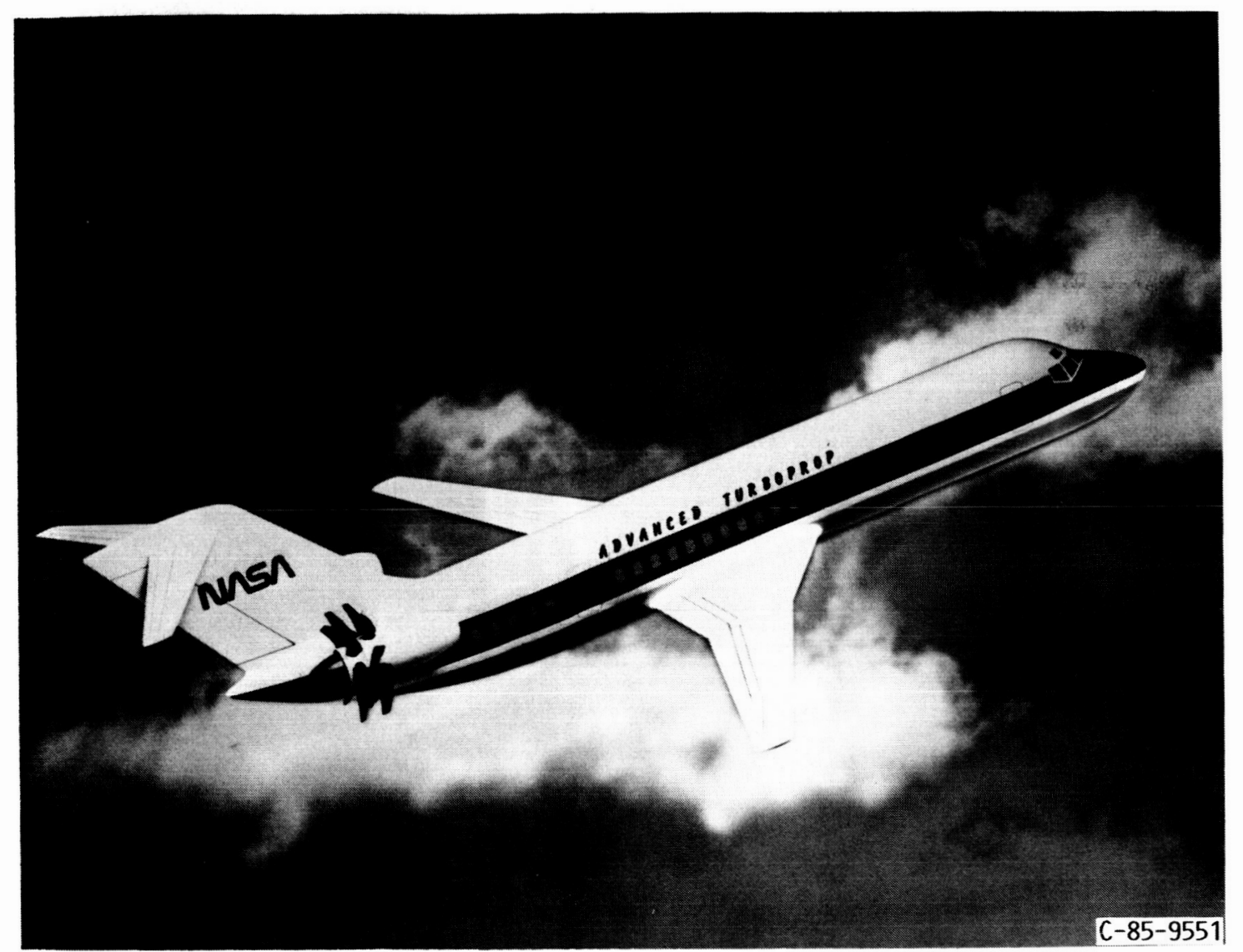

FIGURE 4. - G.E./NASA UDF AFT-MOUNTED PUSHER ENGINE INSTALLATION. 


\section{ORUGWAL PAGE is \\ OF POOR QUALTY}

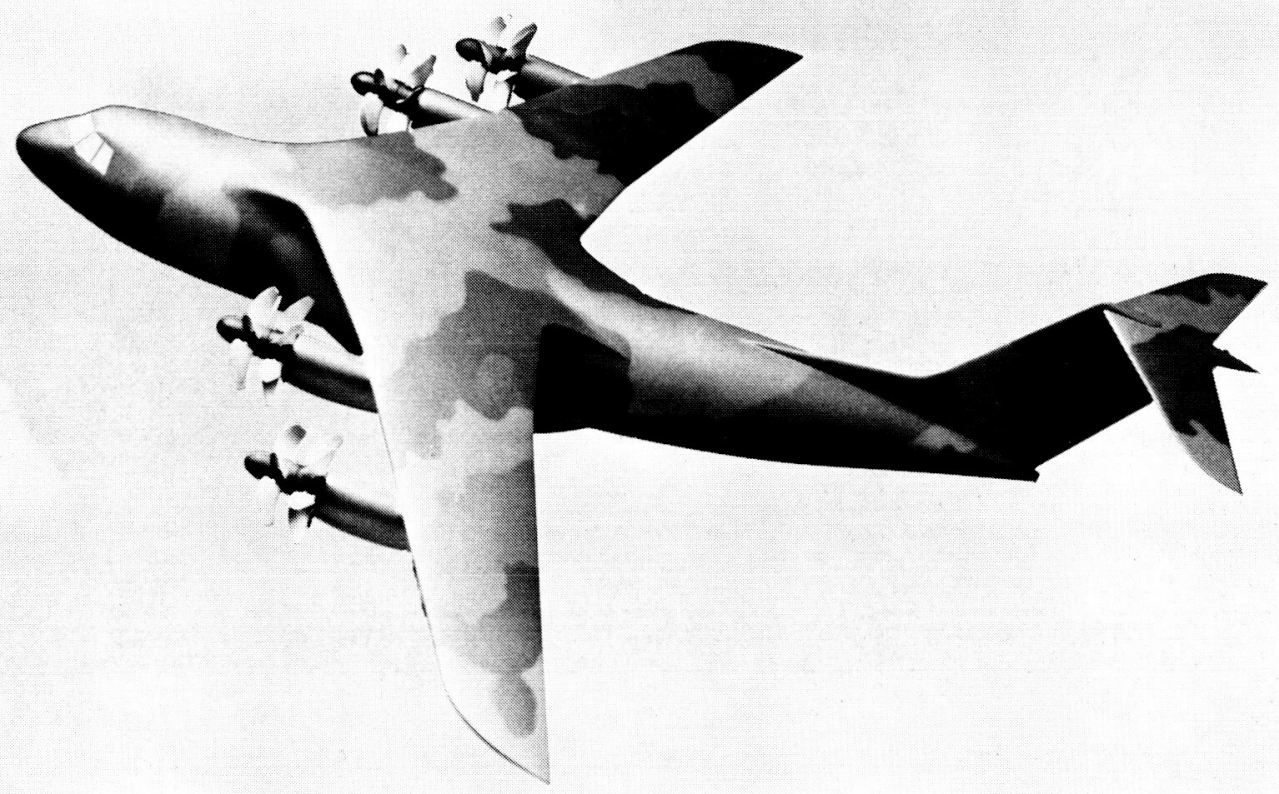

C-86-0139

FIGURE 5. - WING-MOUNTED CR TRACTOR PROPELLER SYSTEM INSTALLATION.

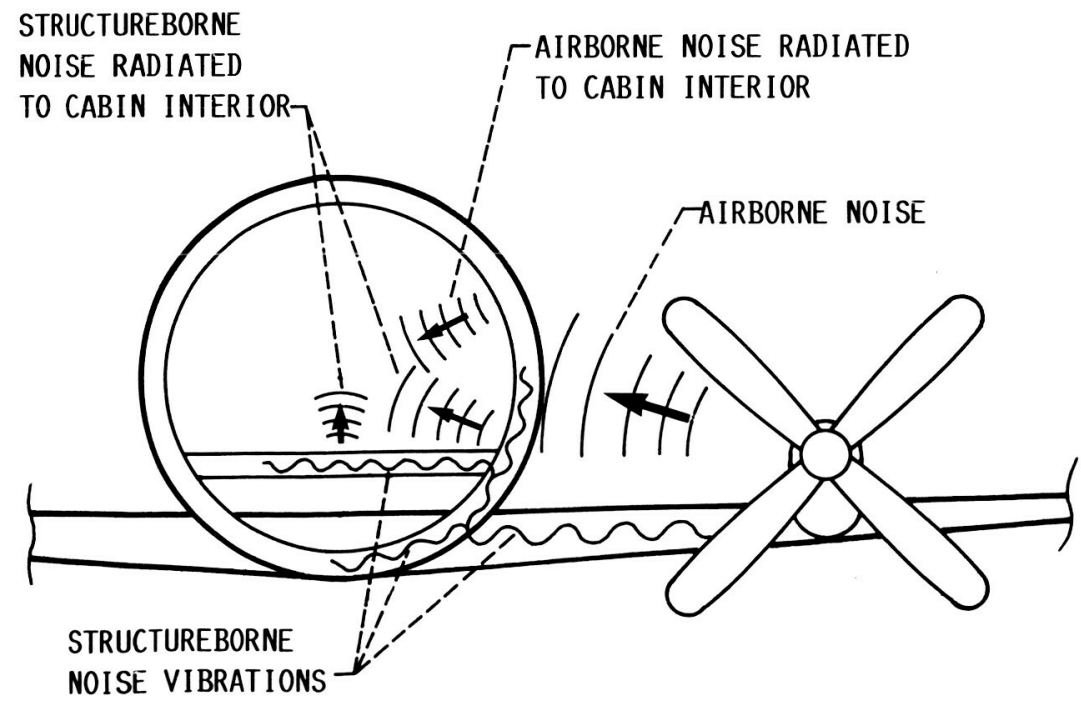

FIGURE 6.- PROPAGATION OF AIRBORNE NOISE AND STRUCTUREBORNE NOISE TO THE CABIN INTERIOR. 


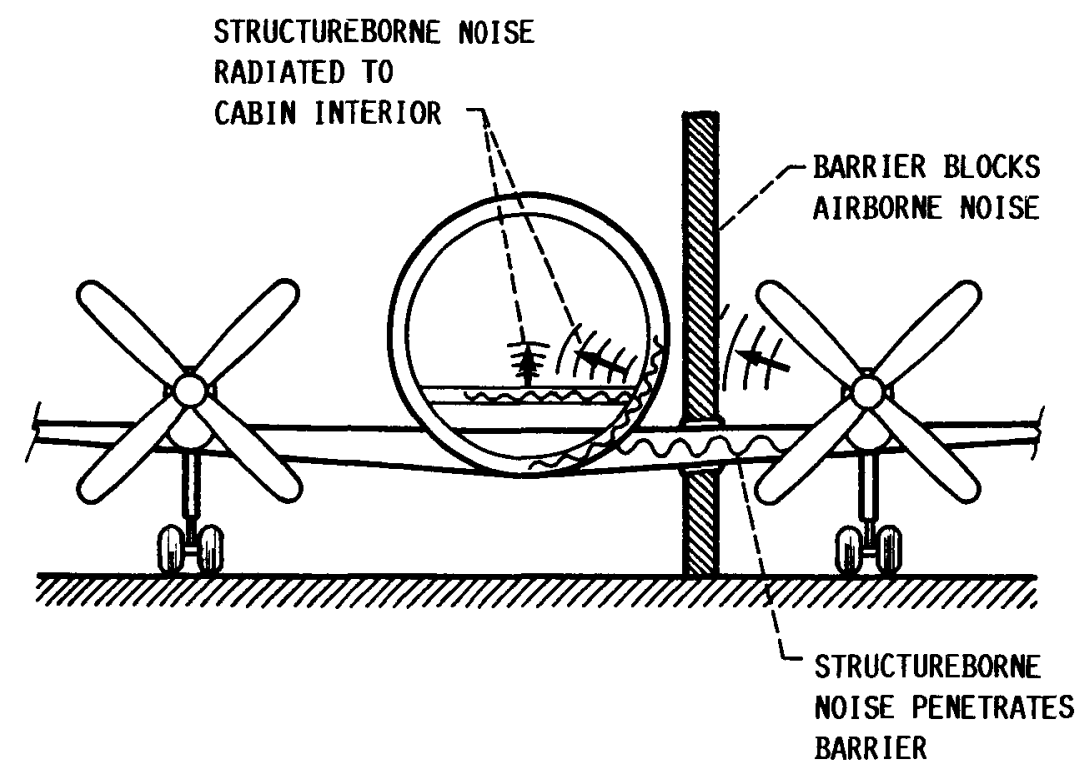

FIGURE 7.- STRUCTUREBORNE MOISE ISOLATED BY MEANS OF AIRBORME NOISE BARRIER IN GROUND TEST.

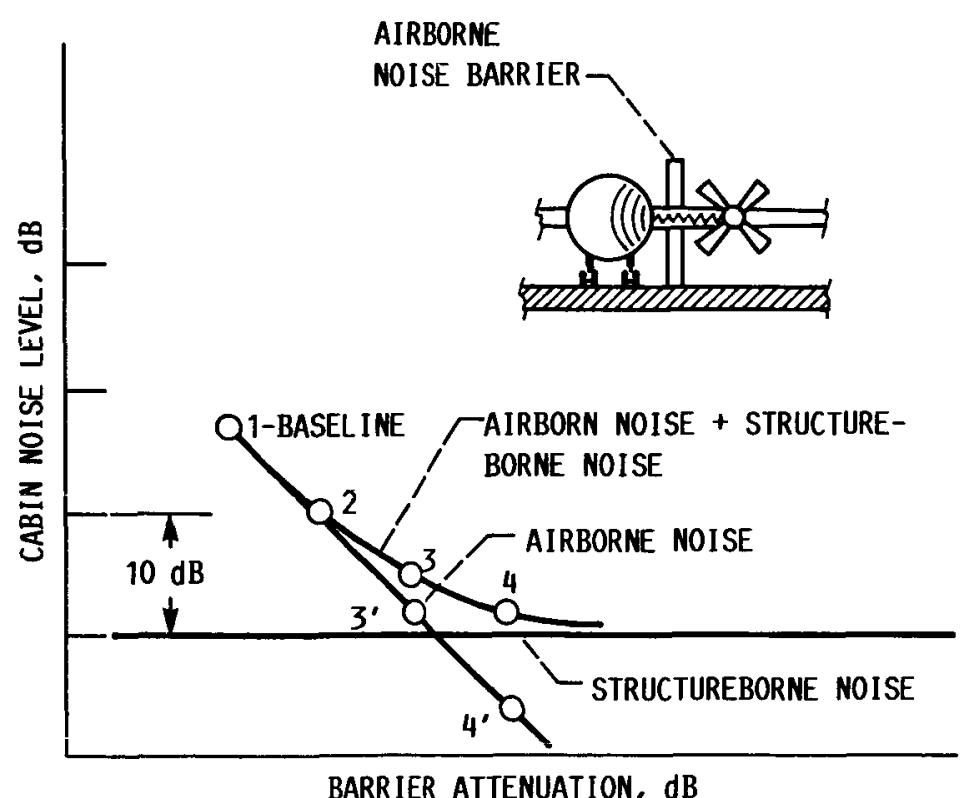

FIGURE 8. - VARIATION OF CABIN NOISE LEVEL WITH VARYING BARRIER TREATMENT. 

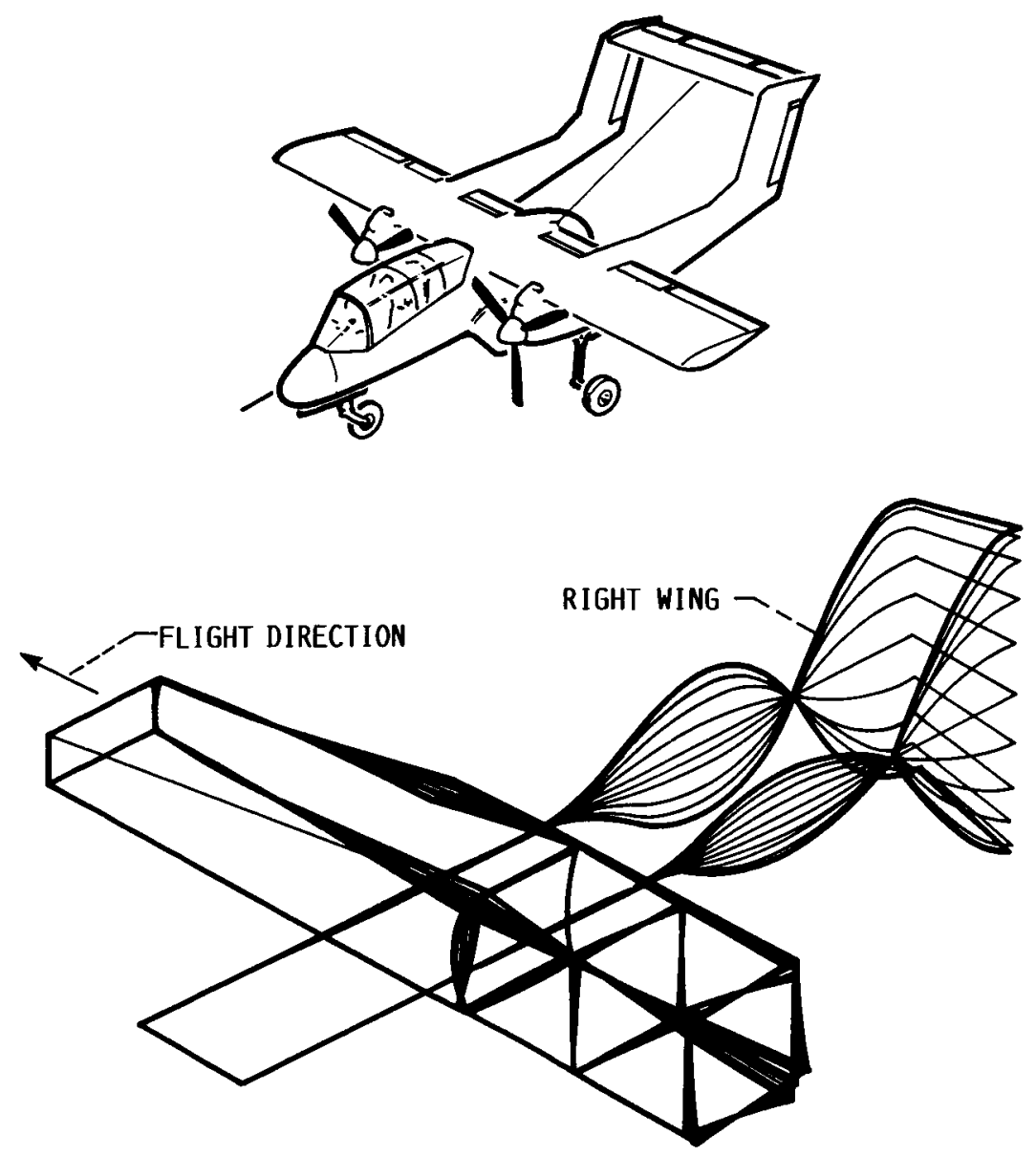

FIGURE 9. - WING AND FUSELAGE VIBRATION MODE AT $201.5 \mathrm{HZ}$ WITH WHITE NOISE SHAKER EXCITATION ON RIGHT WING.

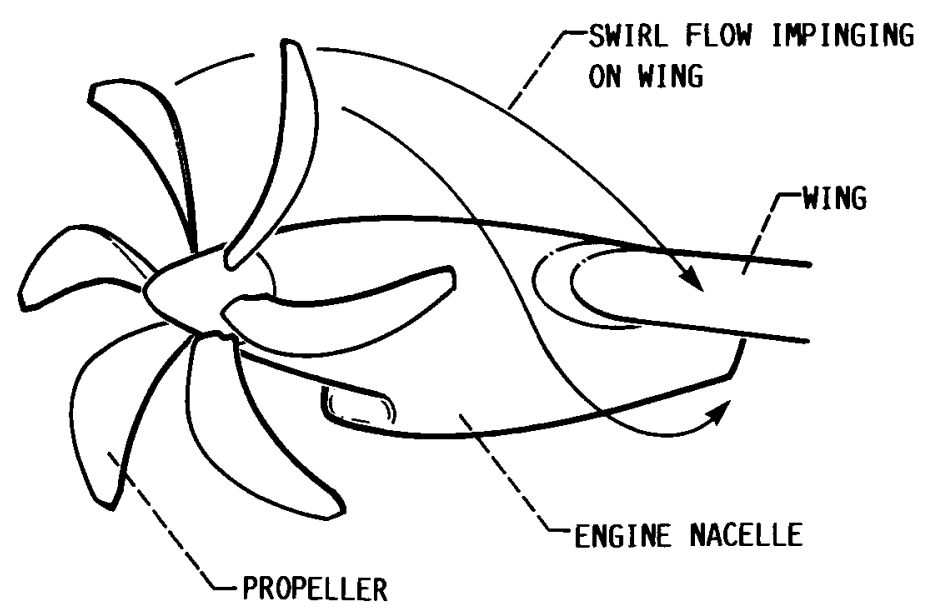

FIGURE 10.- SWIRL FLOW FROM PROPELLER IMPINGING ON DOWNSTREAM WING SURFACES. 


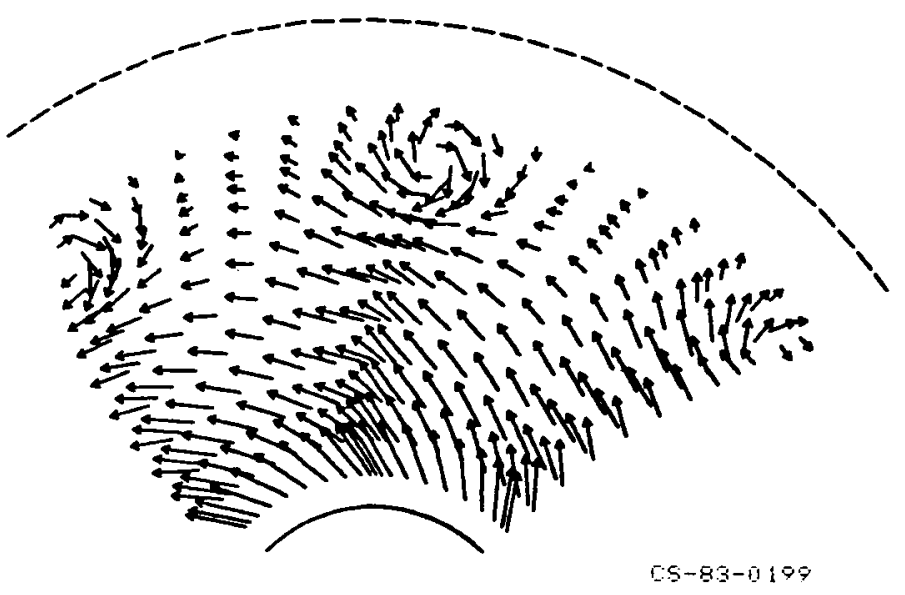

FIGURE 11.- PROPELLER WAKE SECONDARY FLOW.

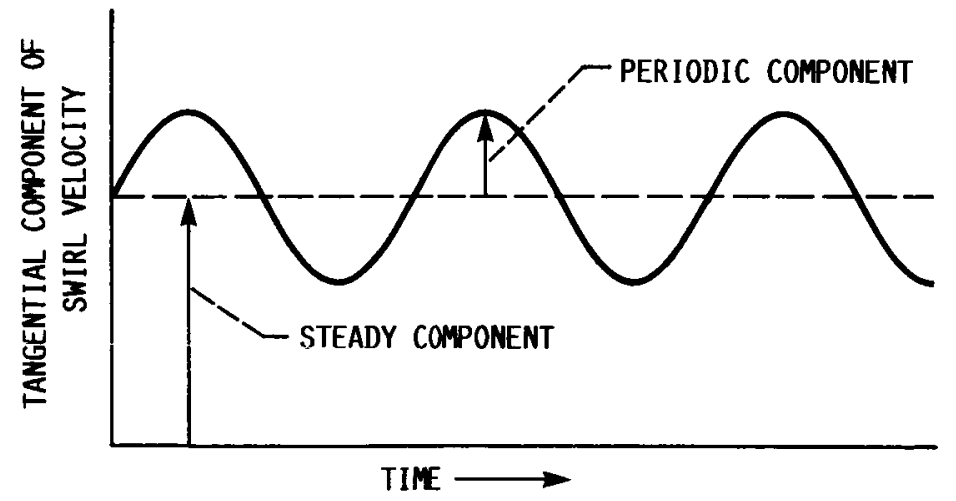

FIGURE 12.- PERIODIC AND STEADY COMPONENTS OF PROPELLER SWIRL VELOCITY. 


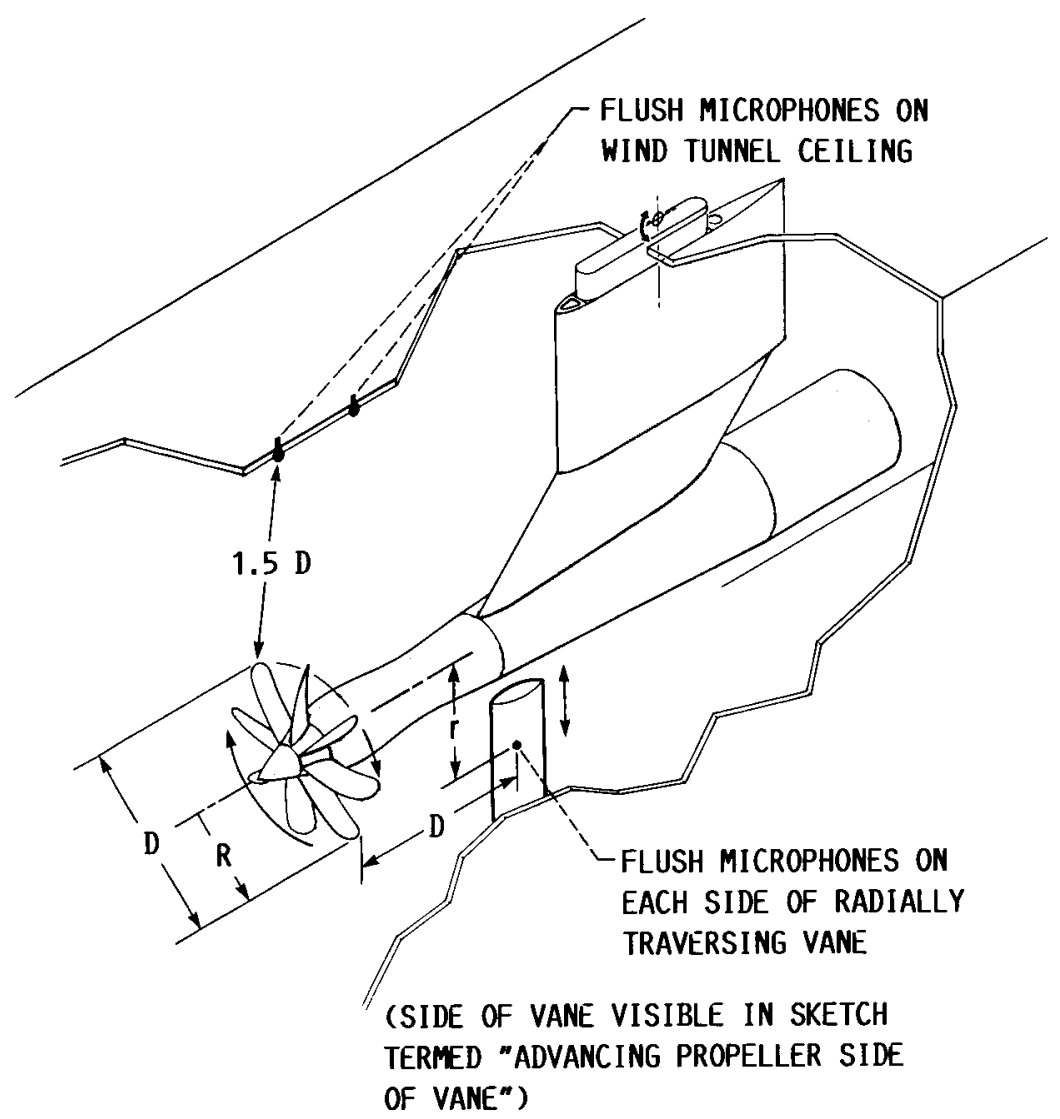

FIGURE 13.- INSTALLATION OF PROPELLER, RADIALLY TRAVERSING VANE, AND MICROPHONES IN WIND TUNNEL.

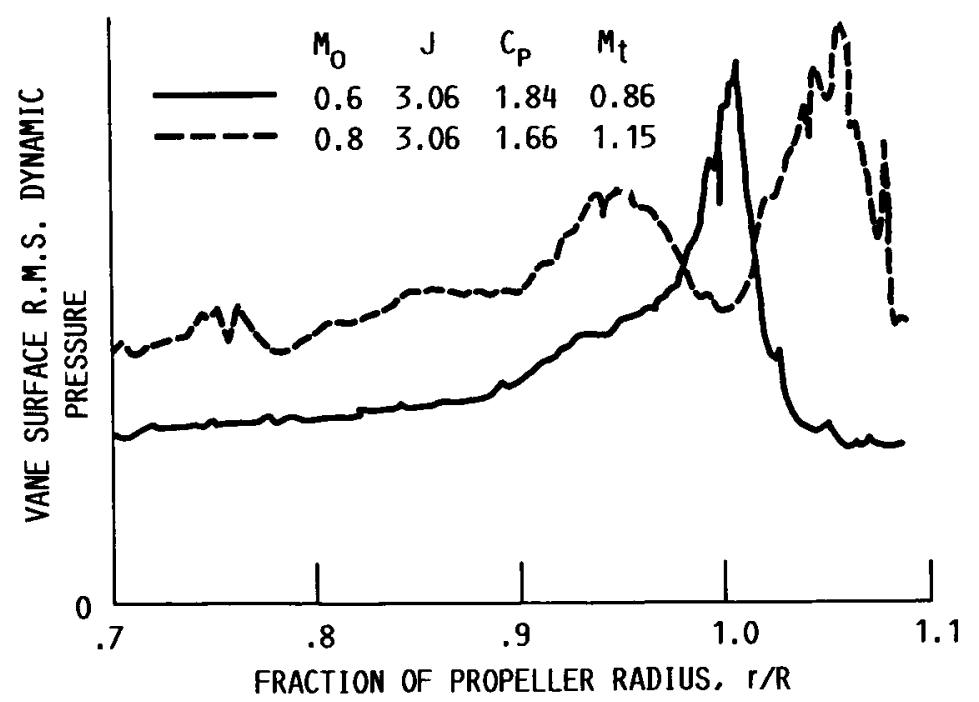

FIGURE 14. - RADIAL VARIATION OF VANE SURFACE R.M.S. DYNAMIC PRESSURE MEASURED IN PROPELLER WAKE, ADVANCING PROPELLER SIDE OF VANE. 


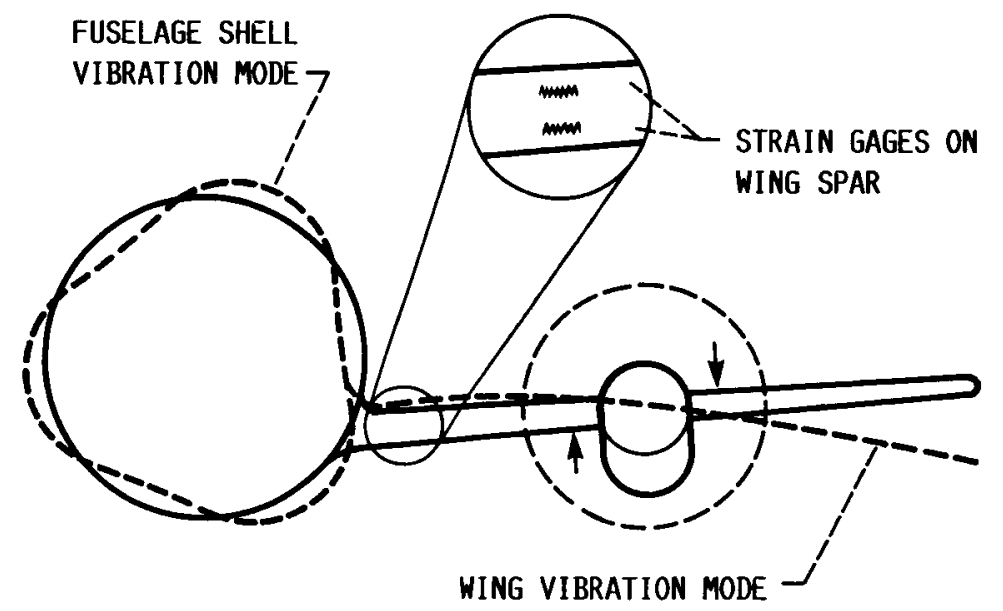

FIGURE 15. - WING AND FUSELAGE VIBRATION MODES DUE TO PROPELLER WAKE PERIODIC TORQUE LOAD.

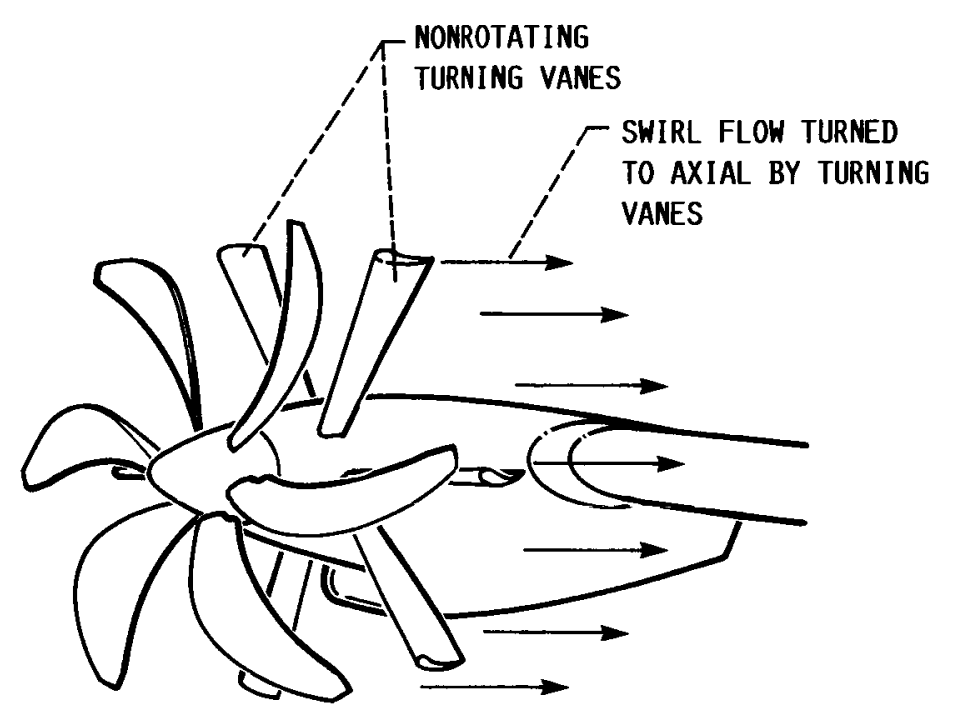

FIGURE 16.- SWIRL FLOW TURNED TO AXIAL BY NONROTATING TURNING VANES. 

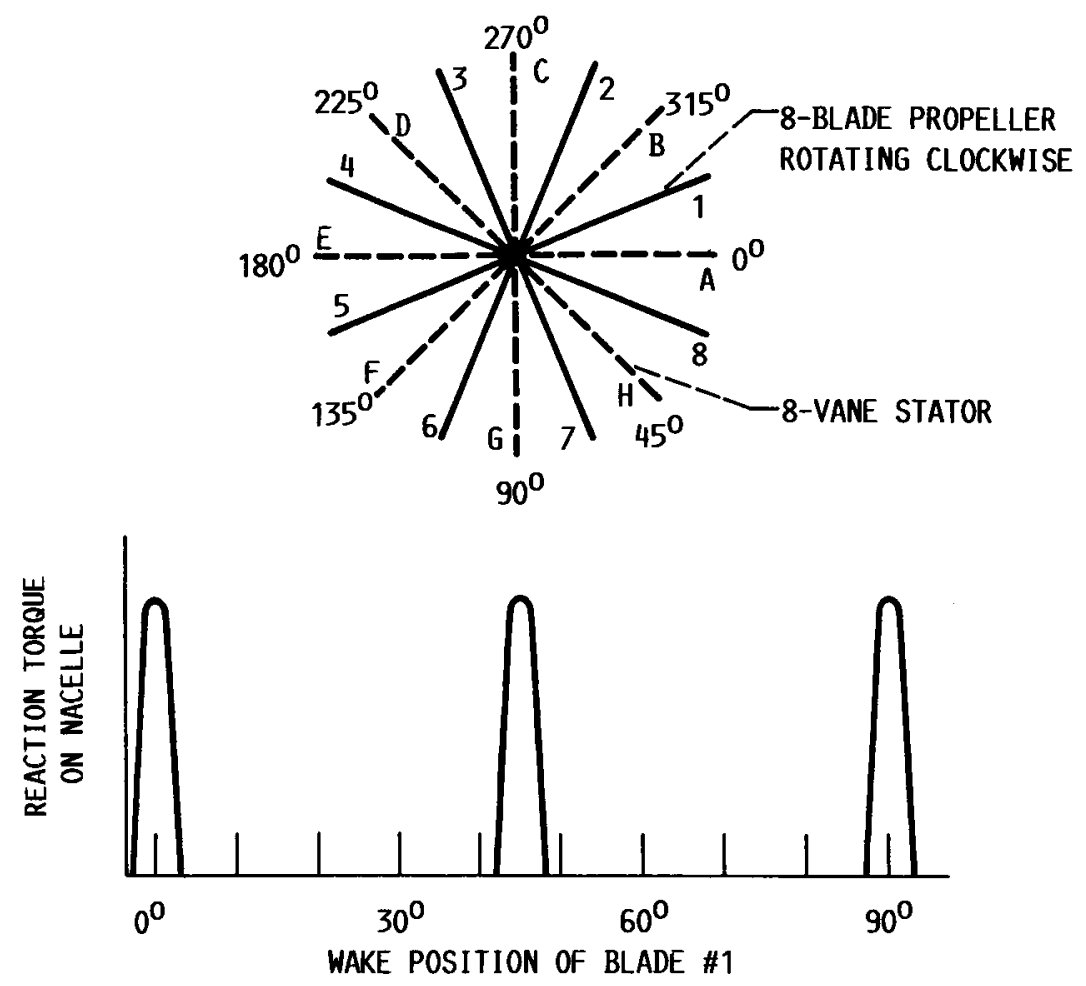

FIGURE 17.- REACTION TORQUE ON NACELLE FOR 8-BLADE PROPELLER WITH 8-VANE STATOR.
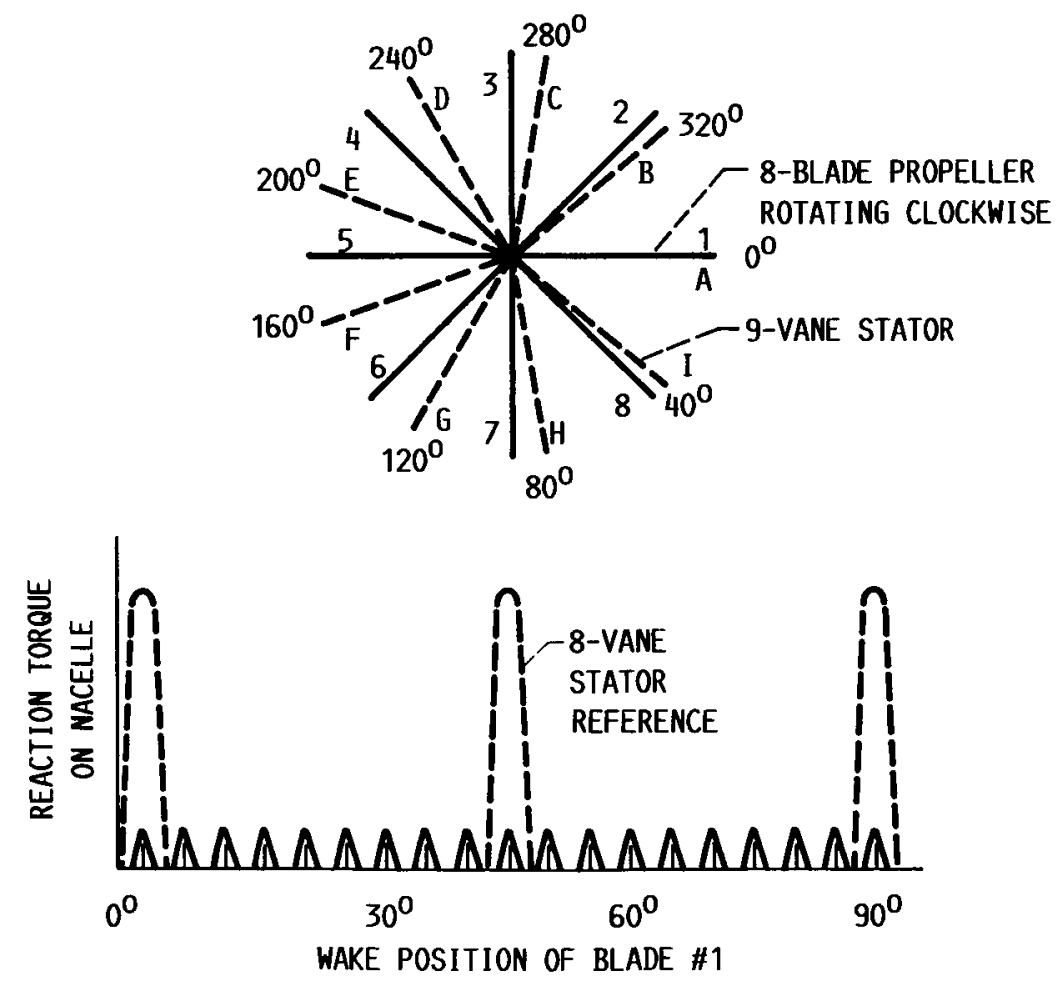

FIGURE 18.- REACTION TORQUE ON NACELLE FOR 8-BLADE PROPELLER WITH 9-VANE STATOR. 


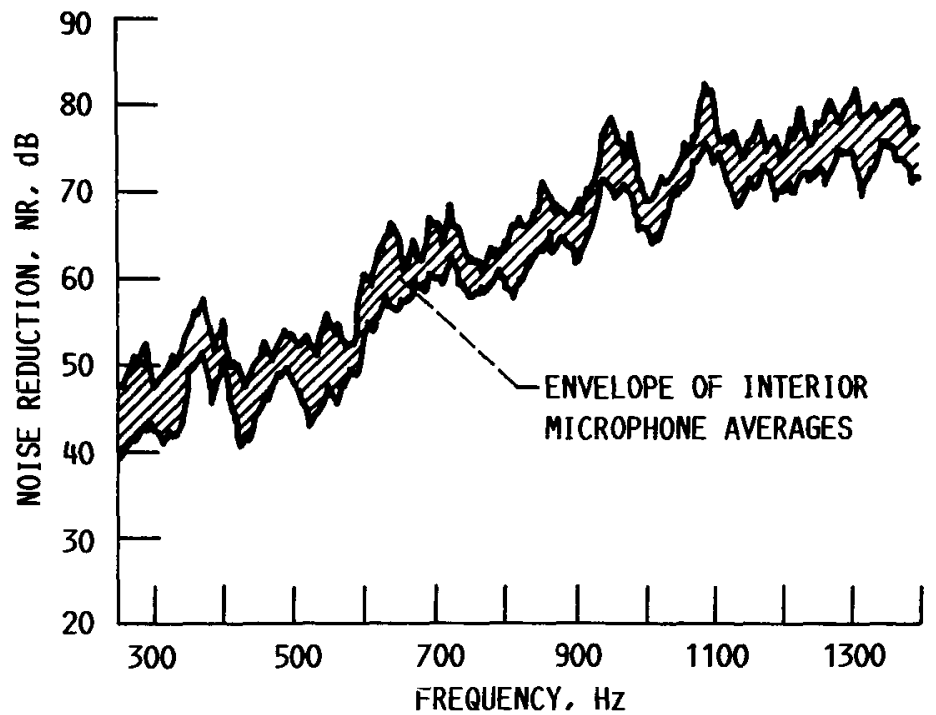

FIGURE 19.- MEASURED NOISE REDUCTION FOR AN EXPERIMENTAL DOUBLE-WALL ACOUSTIC TREATMENT FOR ADVANCED TURBOPROP AIRCRAFT. 


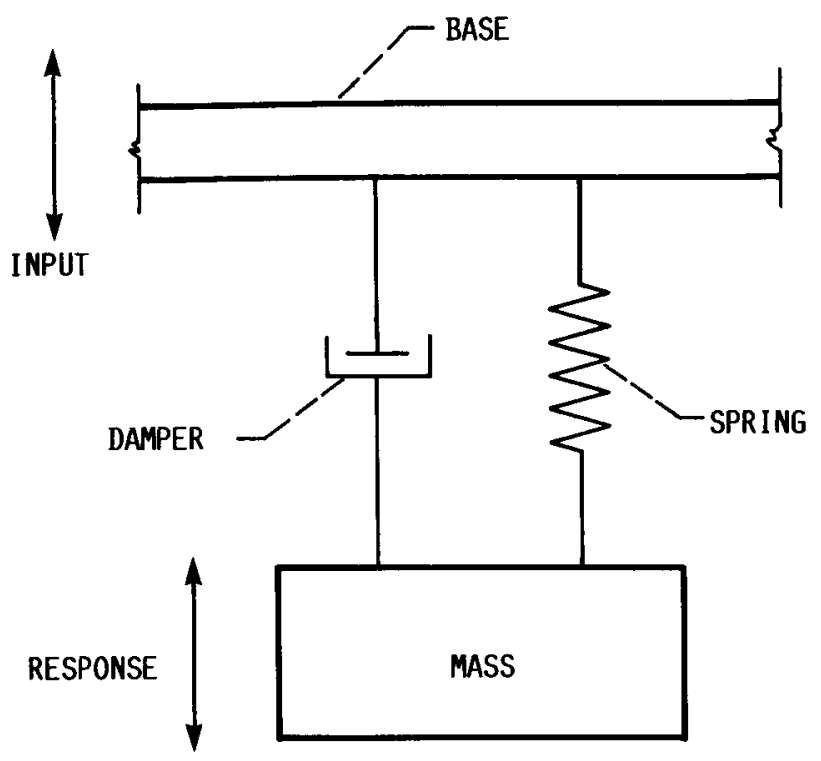

(A) IDEAL SYSTEM.

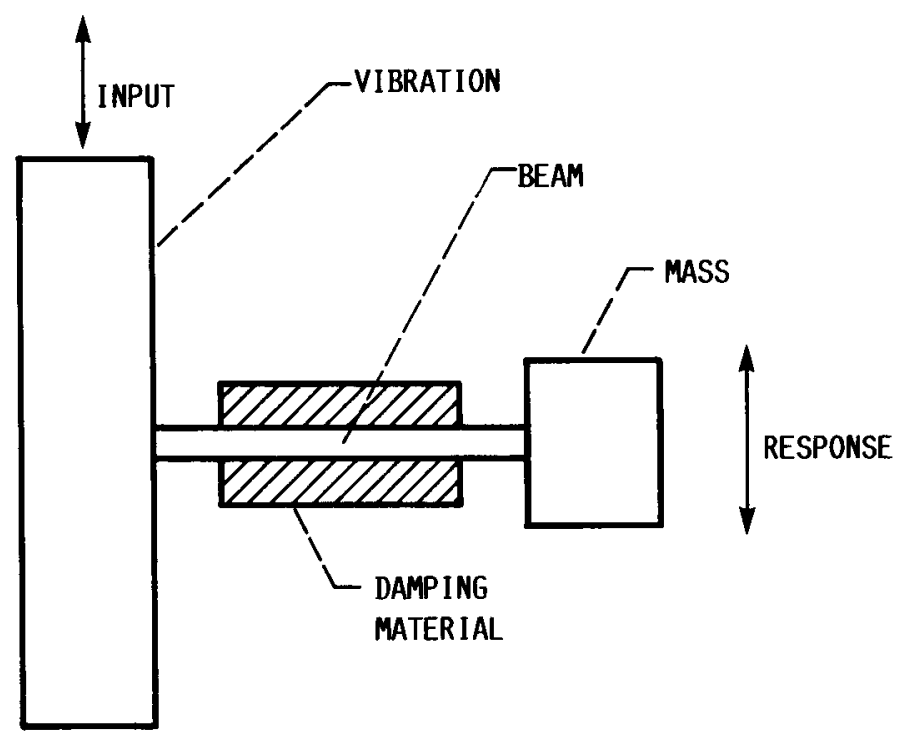

(B) TYPICAL PHYSICAL ARRANGEMENT.

FIGURE 20.- TUNED MECHANICAL ABSORBER. 


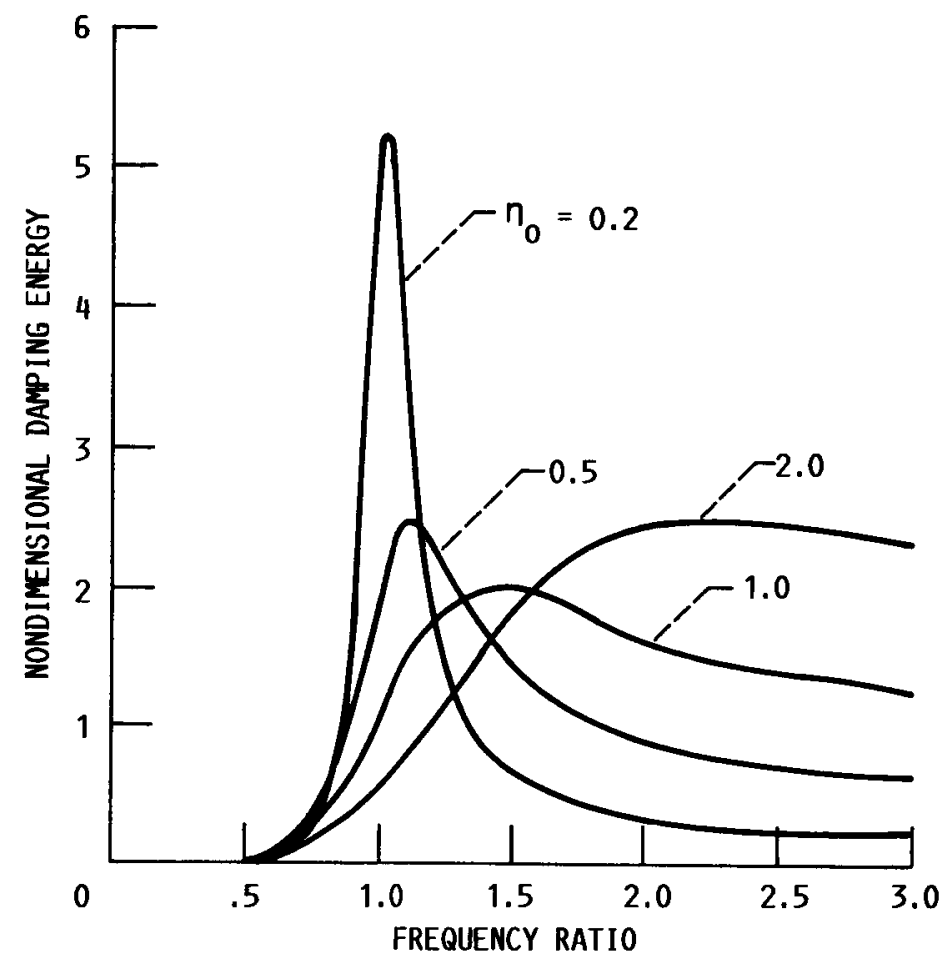

FIGURE 21. - VIBRATIONAL ENERGY DISSIPATION OF TUNED MECHANICAL ABSORBER. 


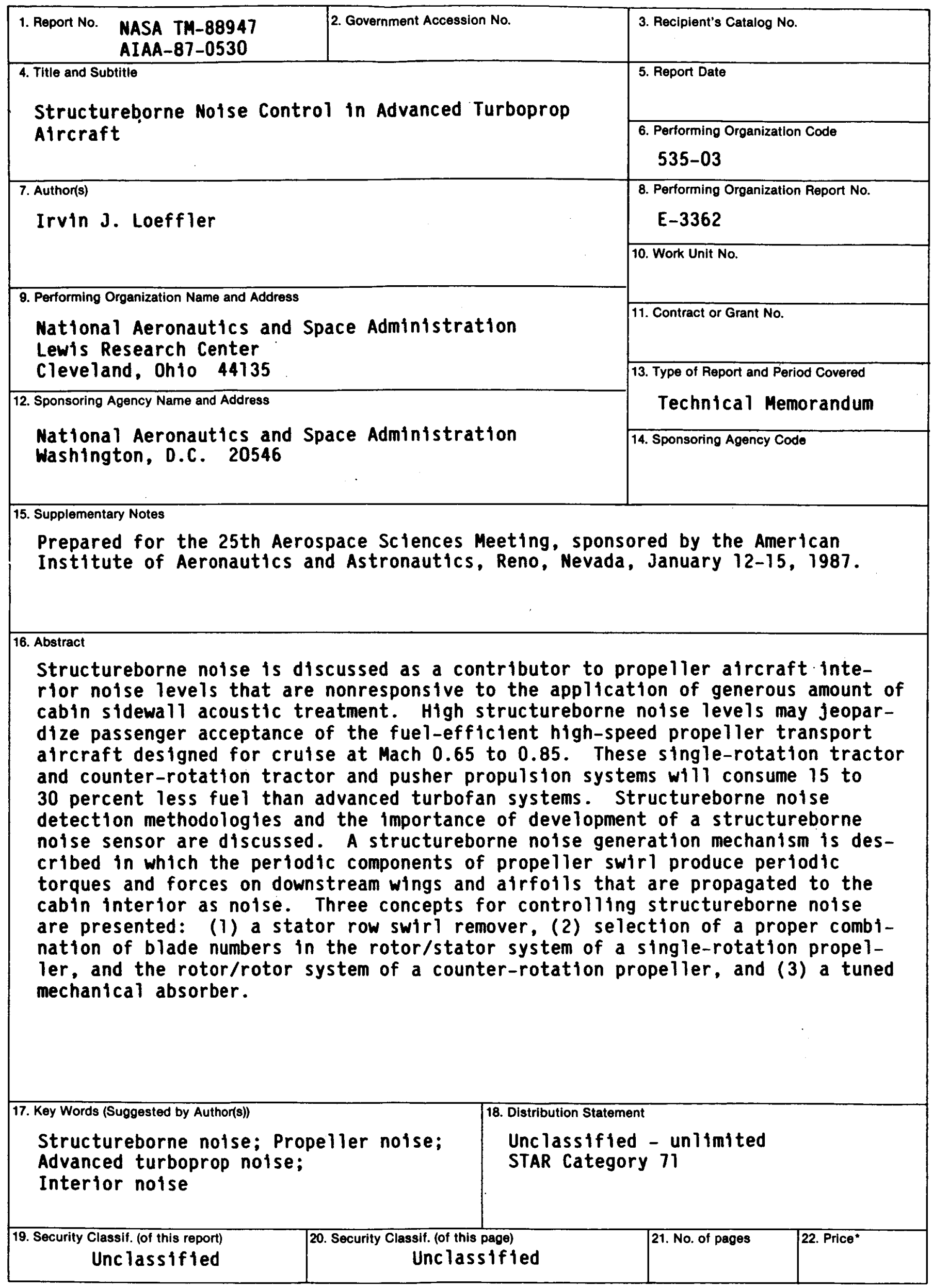

"For sale by the National Technical Information Service, Springfield, Virginia 22161 